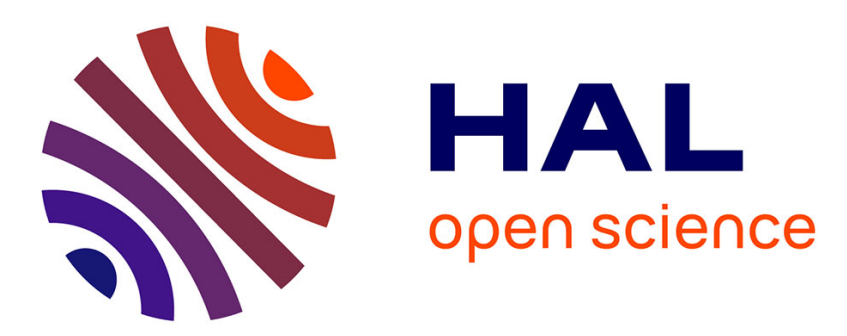

\title{
What else does your biometric data reveal? A survey on soft biometrics
}

Antitza Dantcheva, Petros Elia, Arun Ross

\section{To cite this version:}

Antitza Dantcheva, Petros Elia, Arun Ross. What else does your biometric data reveal? A survey on soft biometrics. IEEE Transactions on Information Forensics and Security, 2015, 11 (3), pp.441 - 467. 10.1109/TIFS.2015.2480381 . hal-01247885

\section{HAL Id: hal-01247885 \\ https://inria.hal.science/hal-01247885}

Submitted on 22 Dec 2015

HAL is a multi-disciplinary open access archive for the deposit and dissemination of scientific research documents, whether they are published or not. The documents may come from teaching and research institutions in France or abroad, or from public or private research centers.
L'archive ouverte pluridisciplinaire HAL, est destinée au dépôt et à la diffusion de documents scientifiques de niveau recherche, publiés ou non, émanant des établissements d'enseignement et de recherche français ou étrangers, des laboratoires publics ou privés. 


\title{
What Else Does Your Biometric Data Reveal? A Survey on Soft Biometrics
}

\author{
Antitza Dantcheva, Petros Elia, Arun Ross
}

\begin{abstract}
Recent research has explored the possibility of extracting ancillary information from primary biometric traits, viz., face, fingerprints, hand geometry and iris. This ancillary information includes personal attributes such as gender, age, ethnicity, hair color, height, weight, etc. Such attributes are known as soft biometrics and have applications in surveillance and indexing biometric databases. These attributes can be used in a fusion framework to improve the matching accuracy of a primary biometric system (e.g., fusing face with gender information), or can be used to generate qualitative descriptions of an individual (e.g., "young Asian female with dark eyes and brown hair"). The latter is particularly useful in bridging the semantic gap between human and machine descriptions of biometric data. In this paper, we provide an overview of soft biometrics and discuss some of the techniques that have been proposed to extract them from image and video data. We also introduce a taxonomy for organizing and classifying soft biometric attributes, and enumerate the strengths and limitations of these attributes in the context of an operational biometric system. Finally, we discuss open research problems in this field. This survey is intended for researchers and practitioners in the field of biometrics.
\end{abstract}

Index Terms-Soft biometrics, Biometrics, Computer Vision, Gender, Age, Ethnicity, Race, Cosmetics, Privacy, Semantics, Visual Attributes

\section{INTRODUCTION}

\section{A. Biometrics}

Biometrics is the science of recognizing individuals based on their physical, behavioral, and physiological attributes such as fingerprint, face, iris, gait and voice [111]. A classical biometric system acquires biometric data from an individual (e.g., a fingerprint image), extracts a set of features from the data, and compares this feature set with templates in the database in order to verify a claimed identity or to determine an identity.

While biometric data is typically used to recognize individuals, it is possible to deduce other types of attributes of an individual from the same data. For example, attributes such as age, gender, ethnicity, height, hair color and eye color can be deduced from data collected for biometric recognition purposes. Recent work [270] has established the possibility

Copyright (c) 2013 IEEE. Personal use of this material is permitted. However, permission to use this material for any other purposes must be obtained from the IEEE by sending a request to pubs-permissions@ieee.org

During the preparation of this manuscript, A. Dantcheva was supported as a Postdoctoral Fellow at West Virginia University and Michigan State University, and as an ERCIM "Alain Bensoussan" Fellow at INRIA funded by the European Union Seventh Framework Programme (FP7/2007-2013) under grant agreement \# 246016. A. Ross was funded in part by US NSF CAREER Award \# IIS 0642554.

A. Dantcheva is with the STARS team of Inria, France. A. Ross is with the Department of Computer Science and Engineering, Michigan State University. P. Elia is with the Mobile Communications Department at Eurecom, France. E-mail: Antitza.Dantcheva@inria.fr, elia@eurecom.fr, rossarun@cse.msu.edu of computing the body mass index (BMI) from face images, thereby suggesting the possibility of assessing health from biometric data.

\section{B. Soft Biometrics}

These additionally deduced attributes, while not necessarily unique to an individual, can be used in a variety of applications. Further, they can be used in conjunction with primary biometric traits in order to improve or expedite recognition performance.

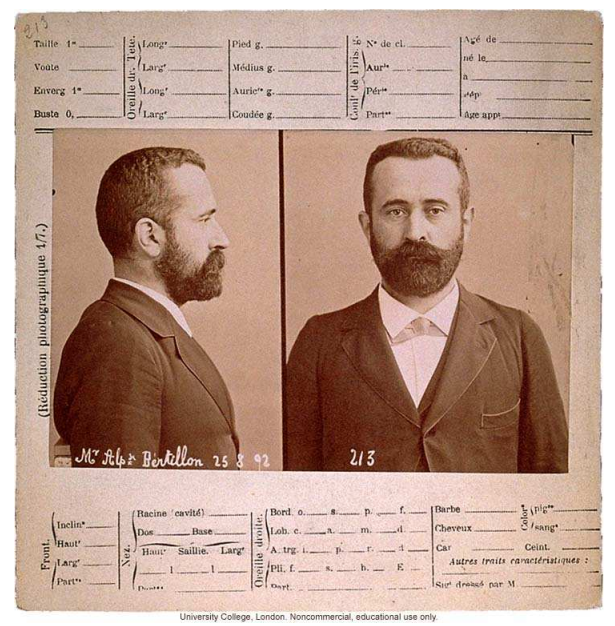

Fig. 1. Anthropometry card of Alphonse Bertillon, who originated the criminal identification system based on profile and full-face photos, and key body measurements (1892). These key measurements include body height, body weight, build, complexion, head length, head width, cheek width, measurements of right ear and left foot, as well as "peculiar marks" such as birthmarks, scars, and tattoos.

It is perhaps this latter application that has led to these attributes being referred to as soft biometrics [109], [110], [180] or light biometrics [4]. In this context, soft biometrics can be traced back to Bertillon [211] (see Figure 1), who brought to the fore the idea of using anatomical, morphological and anthropometrical characteristics for person identification. These attributes have also been referred to as semantics [223], [207], in reference to their semantic interpretation (e.g., describing a face as "young male").

1) Scope and benefits: Various researchers have attempted to define the scope of soft biometrics. Jain et al. [109] defined soft biometrics to be the set of characteristics that provide some information for recognizing individuals, but that are not capable of distinguishing between individuals, mainly due to their lack of distinctiveness and permanence. Samangooei et al. [223], as well as Reid and Nixon [208], further associated soft 
biometrics with labels which people use to describe each other: an association that nicely bridges the gap between human and machine descriptions of biometric data.

Combining the above with the ideas in Dantcheva et al. [42], and keeping in mind that such soft traits can go beyond person recognition, one could define soft biometrics as follows. Soft biometric traits are physical, behavioral, or material accessories, which are associated with an individual, and which can be useful for recognizing an individual. These attributes are typically gleaned from primary biometric data, are classifiable in pre-defined human understandable categories, and can be extracted in an automated manner.

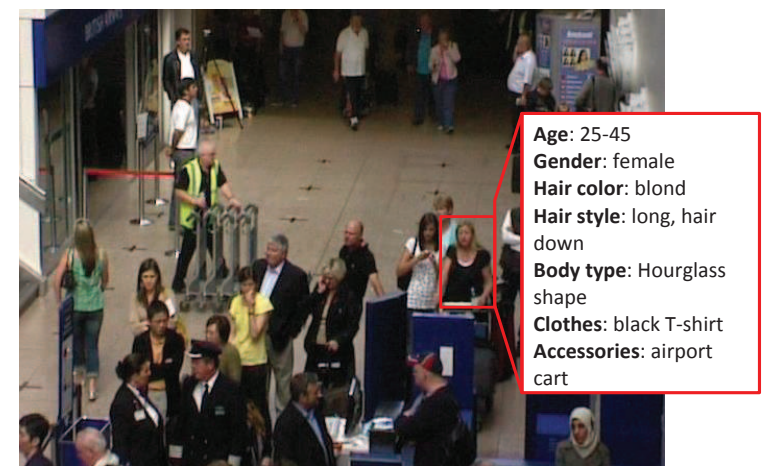

Fig. 2. Importance of soft biometrics. Typical video surveillance scenario: when faces are of low resolution, appear in different poses, and are either occluded or not visible, other attributes such as age, gender, hair color and style, height, body type, clothes and accessories can be used for identification and re-identification. Image obtained from PETS 2007 [63].

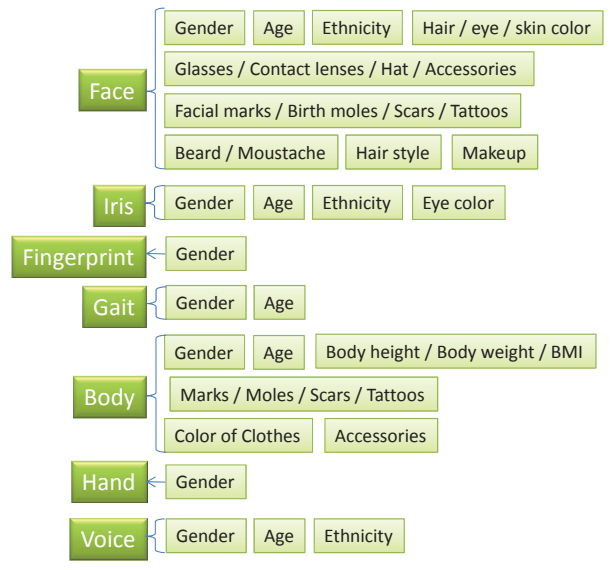

Fig. 3. Ancillary information, referred to as soft biometrics, can be gleaned from the following biometric modalities: face, iris, fingerprint, gait, body, hand, voice.

a) Benefits of soft biometrics: Soft biometrics are often descriptive and have a semantic representation. In addition as noted by Jain et al. [110] - they can be inexpensive to compute, discerned at a distance in a crowded environment, and require less or no cooperation of the observed subjects. To elaborate, we note the following benefits.

Human understandable interpretation: Soft biometric attributes have a semantic interpretation, in the sense that that they can provide a description that can be readily understood by humans; for example the description "young, tall, female". This makes them particularly useful in applications such as video surveillance, where they are directly compatible with how humans perceive their surroundings [210], [67], [43], [204], [47], [257], [45], [46]. In other words, when a human attempts to verbally describe a person, obvious characteristics regarding the person's appearance such as gender, age, height and clothes color are often used (e.g., in police reports). This allows soft biometrics to be used in applications where traditional biometrics may be insufficient, as is argued, for example, by Klontz and Jain [125] in the case of the 2013 Boston bombings.

Robustness to low data quality: Some soft biometric attributes can be deduced from low-quality biometric data (see Figure 2. In this context, such attributes can be extracted, when primary biometric data is not conclusive, due to poor acquisition quality. For example, if the input iris image is of poor quality, one could utilize the surrounding periocular information to perform recognition, rather than relying on the iris itself.

Consent-free acquisition: Soft biometrics can often be captured without the consent and cooperation of the observed subject. For example, information about a person's height or gender can be deduced from a distance.

Privacy: Since soft biometric traits are not distinctive, they only provide a partial description of a person (such as "female, tall, young"). This limitation has positive privacy ramifications when it comes to extracting and storing such soft biometric data.

2) Taxonomy: With the aforementioned scope and benefits in mind, it is worth identifying a taxonomy that can facilitate organization and categorization of soft-biometric traits. This taxonomy is based on utility, and it considers four groups of attributes: demographic, anthropometric, medical, and material and behavioral attributes. This categorization - and the more refined sub-categorization based on the modalities of face, iris, body, gait, fingerprint and hand (Figure 3) - will also help us structure the exposition of the state-of-art in the rest of this survey paper.

TABLE I

SOFT BIOMETRIC TAXONOMY WITH FOUR GROUPS: I) DEMOGRAPHIC, II) ANTHROPOMETRIC AND GEOMETRIC, III) MEDICAL, IV) MATERIAL AND BEHAVIORAL.

\begin{tabular}{|c||c|}
\hline Demographic attributes & $\begin{array}{c}\text { age, gender, ethnicity } \\
\text { eye-, hair-, skin-color }\end{array}$ \\
\hline $\begin{array}{c}\text { Anthropometric } \\
\text { and geometric attributes }\end{array}$ & $\begin{array}{c}\text { body geometry } \\
\text { and facial geometry }\end{array}$ \\
\hline Medical attributes & $\begin{array}{c}\text { health condition, BMI/ } \\
\text { body weight, wrinkles }\end{array}$ \\
\hline $\begin{array}{c}\text { Material and behavioral } \\
\text { attributes }\end{array}$ & $\begin{array}{c}\text { Hat, scarf, bag, clothes, } \\
\text { lenses, glasses }\end{array}$ \\
\hline
\end{tabular}

The above taxonomy need not necessarily result in disjoint groups and it is certainly not a unique taxonomy. For example, taxonomies in Jain et al. [110] and Dantcheva et al. [42] (see also [39]) have different definitions that categorize traits based on their ability to distinguish between individuals as well as 
their variability over time.

\section{Domains of application}

Automated soft biometric extraction has a number of applications: in the area of security where algorithms can locate a person-of-interest based on a specific set of soft biometric attributes; in image-tagging and video indexing where photo or video album management can be performed based, for example, on age, gender, and clothing; in human-computerinteraction where data and personalized avatars can be automatically designed according to the user's external appearance (e.g. hair- and skin-color, age and gender); in forensics where artists can amend sketches of the suspect or the victim based on old pictures; and in surveillance where suspects can be located based on semantic descriptions. Other applications include age-specific access control where, for example, children can be prevented from watching certain movies, accessing certain web sites, or entering bars or liquor-stores. There are industrial systems ${ }^{1}$ that extract demographic information of customers for customizing advertisements or for collecting aggregate data about consuming habits (e.g., based on age, gender, ethnicity). In addition, Electronic Customer Relationship Management (ECRM) can use soft biometrics-based categorization for effectively managing customers by offering customized products and services. For example, age or gender specific advertisement can be presented for consumer goods such as mobile phones, fashion, and food. In cosmetology, it is of interest to estimate the rejuvenating effect of decorative cosmetics and cosmetic surgery by computing the perceived age of an individual from their face image.

In video retrieval systems [93], [252], [258], [190], soft biometric traits can be used to locate specific individuals in a video stream either by verbal descriptions (e.g., "individual with a red shirt") or by automatically extracting soft biometric features from an input image and using these features to locate a matching individual in the video stream.

Finally, in health monitoring, soft biometrics are envisioned to play a major role in early diagnosis of illness, sickness prevention and health maintenance. Such traits include body weight / body mass index, skin abnormalities, and wrinkles. We will expand on this possibly later on in the paper.

Below, we describe the various contexts in which soft biometric traits can be used.

1) Uni-modal system: Often applications might require the extraction of a single soft biometric trait (e.g. gender in a gender-personalized advertising campaign), in a so called unimodal soft biometric system. Such a system generally contains the "preprocessing", "feature extraction" and "classification" modules, with the main focus being on the choice of representation (feature extraction).

2) Fusion with primary biometric traits: Here, the goal is to improve the recognition accuracy of a biometric system. Such an approach was proposed by Jain et al. [110], who considered a hybrid system that combined fingerprint identification with soft biometric attributes such as age, gender and height, to improve the overall matching accuracy.

\footnotetext{
${ }^{1}$ http://www.quividi.com/
}

Let $W=\left\{w_{1}, w_{2}, \ldots, w_{n}\right\}$ be the set of $n$ subjects enrolled in the database, and let $\mathbf{x}$ be the feature vector corresponding to the primary biometric system. The output of the primary biometric system is of the form $P\left(w_{i} \mid \mathbf{x}\right), i=1,2, \ldots, n$, where $P\left(w_{i} \mid \mathbf{x}\right)$ is the probability that the input data belongs to subject $w_{i}$ given the feature vector $\mathbf{x}$. Let $\mathbf{y}=\left\{y_{1}, y_{2}, \ldots, y_{m}\right\}$ be the soft biometric feature vector. Then the updated probability $P\left(w_{i} \mid \mathbf{x}, \mathbf{y}\right)$ that the subject in question is $w_{i}$, can be calculated using the Bayes rule to be

$$
P\left(w_{i} \mid \mathbf{x}, \mathbf{y}\right)=\frac{p\left(\mathbf{y} \mid w_{i}\right) P\left(w_{i} \mid \mathbf{x}\right)}{\sum_{j=1}^{n} p\left(\mathbf{y} \mid w_{j}\right) P\left(w_{j} \mid \mathbf{x}\right)}
$$

where, $p\left(\mathbf{y} \mid w_{i}\right), i=1,2, \ldots, n$ represents the conditional probability of the random vector $\mathbf{y}$ given subject $w_{i}$.

Other notable research on fusing soft biometrics and classical biometrics, include the works in [229], [112], [109], [1], [289], [189].

3) Search space reduction: Soft biometrics can also be used to expedite the search in large biometric databases by filtering out subjects. A number of attributes such as age, gender, hair and skin color have been proposed for efficient filtering of face databases [129], [130], [103]. Furthermore, an analysis of the filtering-gain versus filtering-reliability tradeoff in using soft biometric traits to prune large databases was presented in [41].

\section{Visual attributes}

The computer vision community refers to describable visual attributes as any visual and contextual information that is helpful in representing an image (cf. Scheirer et al. [229]). In this approach, semantically meaningful labels are employed towards image retrieval and object categorization. In the context of human recognition, this semantic information can describe gender [228], ethnicity [229], accessories [21], clothing style [238], and facial-feature-shapes [228]. Related work include fusion of attributes by Scheirer et al. [227], pruning of large-scale datasets by Russakovsky and Fei-Fei [220], as well as studies on similarities between faces or objects based on relative attributes by Parikh and Grauman [187] and Zhou et al. [297]. Other pertinent literature include [62], [131], [52], [137], [156], [21]. Of specific interest are the "zeroshot" learning approaches, where previously unseen objects are described using attributes of objects encountered in the training set (cf. Parikh and Grauman [187]).

\section{E. Structure of paper}

The survey provides a review of salient techniques for extracting soft biometrics from modalities such as face, body, fingerprint, iris, and voice. While an exhaustive survey of all soft biometric traits is not possible due to the richness of the field (for example, we do not expand on traits relating to the ear, or to saccadic movements), we try to offer a holistic view of most of these traits. In this way, this survey paper is significantly different from other introductory overviews (see [42], [210], [67], [213], [106] and Table II) that have focused on specific soft biometric traits such as gender, age or ethnicity. 
To appear in IEEE Transactions on Information Forensics and Security (TIFS), 2015

The structure of this survey is based on the aforementioned taxonomy of soft biometrics. We discuss soft biometric traits that are heavily used as demographic attributes (Section II), as anthropometric (geometric) attributes (Section III), medical attributes (Section IV), and as miscellaneous material and behavioral attributes (Section V). Finally in Section VI we discuss open research problems that are currently being addressed in the field of soft biometrics.

TABLE II

EXISTING INTRODUCTORY OVERVIEWS ON GENDER AND AGE ESTIMATION TECHNIQUES.

\begin{tabular}{|c|c|c|}
\hline Modality & Scientific work & Year \\
\hline \hline Gender & Ng et al. [177] & 2012 \\
\hline Gender & Khan et al. [120] & 2011 \\
\hline Gender & Bekios-Calfa et al. [18] & 2011 \\
\hline Gender & Ramanathan et al. [201] & 2009 \\
\hline Gender & Mäkinen and Raisamo [157] & 2008 \\
\hline Gender & Mäkinen and Raisamo [158] & 2008 \\
\hline Age & Guo [90] & 2012 \\
\hline Age & Fu et al. [68] & 2010 \\
\hline Ethnicity & Fu et al. [65] & 2014 \\
\hline
\end{tabular}

\section{Demographic ATtRIBUtes}

The term demographics, in addition to referring to the quantifiable statistics of a given population, refers to attributes such as age, gender, ethnicity and race that are widely used in common population statistics. Since the early publications in [164], [243], research on this class of soft biometrics has been embraced by the computer vision community.

\section{A. Gender Estimation}

The traditional definition of sex refers to the biological characteristics that differentiate men and women, as opposed to gender, which is related to the social and cultural distinctions between the sexes. However, very often, the terms "sex" and "gender" have been used interchangeably in the biometrics literature. Consequently, we do not make any explicit distinction between the two terms in this article.

Gender estimation remains a challenging task, which is inherently associated with different biometric modalities including fingerprint, face, iris, voice, body shape, gait, signature, DNA, as well as clothing, hair, jewelry and even body temperature (see [165]). The forensic literature [148] suggests that the skull, specifically the chin and the jawbone, as well as the pelvis, are the most significant indicators of the gender of a person; in juveniles, these shape-based features have been recorded to provide classification accuracy of $91 \%-99 \%$. It has been argued (see for example the work by Loth and Iscan [148]) that there is no single skeletal feature that definitely reveals the evidence of sexual dimorphism, and that there is in fact a cross-gender metric overlap of up to $85 \%$, which can be attributed to environmental influences and pathologic conditions, such as diet and occupational stress. In spite of this, forensic experts argue [128] that near $100 \%$ gender determination accuracy can be attained by visual examination of the entirety of the skeleton.

Humans are generally quite good at gender recognition, as they have been programmed - from an evolutionary standpoint - to classify gender from early on in their lives [185]. As pointed out by Edelman et al. [54], humans perform face image-based gender classification with an error rate of about $11 \%$, which is commensurate to that of a neural network algorithm performing the same task (at that point in time).

Despite this, automated gender recognition from biometric data remains to be a challenge and is impacted by other soft biometrics, for example, age and race; gender dimorphism is accentuated only in adults, and varies across different races.

1) Gender from face: In gender recognition from face, feature-based approaches extract and analyze a specific set of discriminative facial features (patches) in order to identify the gender of a person. This is a particularly challenging problem, as is implied from the fact that female and male average facial shapes are generally found to be very similar.

One of the primary challenges in face-based gender recognition is the step of feature selection, where one must judicially select the type of considered features in order to improve gender recognition rates. Towards understanding this feature selection process, different types of strategies have been attempted, such as the work in Sun et al. [245] that employed genetic algorithms for eigen-feature selection. Other approaches focus on specific facial features, such as the approach by Zhang et al. [292] that focused on the eye brow and the jaw region.

Another challenge comes in unconstrained settings where the face image is affected by changes in illumination, pose, etc. While in more constrained settings face-based gender estimation has been reported to achieve classification rates of up to $99.3 \%$ (see Table III), this performance significantly decreases in more realistic and unconstrained settings.

The majority of gender classification methods contain two steps succeeding face detection, namely feature extraction and pattern classification.

Feature extraction: Notable efforts include the early work by Moghaddam et al. [169] and the work by Baluja et al. [15] who used raw pixel intensities as inputs to SVM and Adaboost classifiers, in order to achieve a $96 \%$ success rate on low resolution images. Interesting work can also be found in Cao et al. [22] who investigated facial metrology for pertinent gender traits, which resulted in error rates that were observed to be between $3.8 \%$ and $5.7 \%$ lower than that of appearancebased methods. Other feature extraction approaches are found in the work of Saatci and Town in [221], who presented an active appearance model (AAM) based geometric-approach for extracting gender and expression (using a SVM classifier with a radial basis kernel), as well as recent approaches that use SIFT [254], LBP [281], [157], semi-supervised discriminant analysis (SDA) [19] or combinations of different features [83], [265].

Classification: A number of classification methods have been used for gender estimation, and a useful comparative guide of these classification methods can be found in Mäkinen and Raisamo [157]. One interesting conclusion of their work 
was that image size did not greatly influence the classification rates. This same work also revealed that manual alignment affected the classification rates positively, and that the best classification rates were achieved by SVM.

The area of gender estimation has also received numerous other contributions such as those that go beyond using static $2 D$ visible spectrum face-images. Related publications include the work of Han et al. [97] that explored the use of 3D face images and SVMs, achieving gender classification with an average error rate of $17.44 \%$ on the GavabDB database that contained multiple facial surface images of 45 males and 16 females. Chen and Ross [27] and Ross and Chen [219] used near-infrared (NIR) and thermal face images for gender classification. Their work demonstrated that the local binary pattern histogram (LBPH)-descriptor and SVM classifier offer the best accuracy results, which were reported to reach $93.59 \%$ for NIR images; an accuracy of $90.66 \%$ was achieved for thermal images using LBP+PCA+SVM. Satta et al. [226] used - in addition to facial features (represented by LBPHPyr (local) and LDA (global) features) - other contextual features such as hair (captured by HSV histograms, fuzzy color and texture histogram (FCTH), and edge histogram descriptor (EHD)) in an AdaBoost framework to obtain an accuracy of about $75 \%$.

2) Gender from Fingerprint: Fingerprint-based gender classification has received attention in forensic anthropology as a pruning tool that can reduce the fingerprint search space by offering a likelihood that a specific fingerprint belongs to a male or a female. This approach exploits the fact that there appear to be differences in male and female fingerprints. Such differences include the ridge-thickness to valley-thickness ratio (RTVTR), the ridge count (the average ridge count is slightly higher in males than in females), and the count of white lines [182].

Gupta and Rao [91] used wavelet transformation and back propagation artificial neural networks to achieve an overall classification rate of $91.45 \%$ on a private database of 550 fingerprints (275 male, 275 female). Similar results were obtained by Badawi et al. [12], who employed Fuzzy Cognitive Maps (FCM) and neural networks to achieve a fingerprintbased gender classification rate of $88 \%$ (see Figure 4). Additionally Tom et al. [255] used 2D wavelet transform and PCA to obtain $70 \%$ accuracy on a 547 subject-database, while Gnanasivam and Muttan [77] fused fingerprint features obtained by discrete wavelet transform (DWT) and singular value decomposition (SVD) to achieve an overall classification rate of $87.52 \%$. Marasco et al. [160] used a combination of image quality and texture features for gender determination with overall classification rates of up to $88.7 \%$ on a dataset of 494 subjects. However, their experimental protocol does not indicate if subjects in the training and test sets were mutually exclusive. Recently, Rattani et al. [205] explored the use of classical texture descriptors - Local Binary Pattern (LBP), Local Phase Quantization (LPQ), Binarized Statistical Image Features (BSIF) and Local Ternary Pattern (LTP) - to estimate gender from fingerprint images and tested them on the WVU fingerprint dataset consisting of 237 users. In their experiments, the subjects used in the training and test sets were mutually exclusive thus resulting in a statistically unbiased evaluation. Fusion of descriptors and different fingers provided overall classification rates of up to $81.4 \%$.

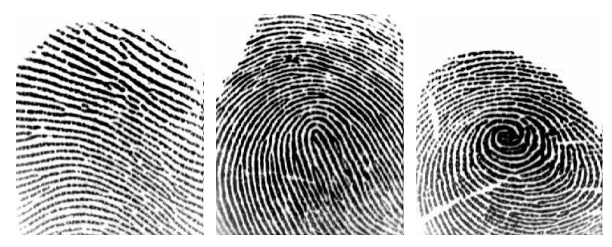

(a)

(b)

(c)

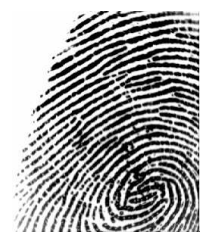

(d)

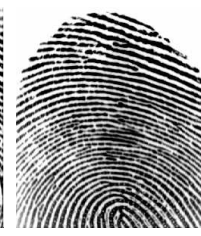

(e)

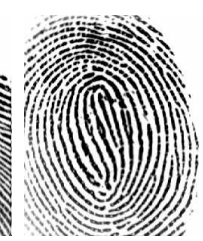

(f)

Fig. 4. Fingerprint patterns obtained from female (first row) and male (second row) subjects [34]. Fingerprint-based gender classification in forensic anthropology considers ridge thickness to valley thickness ratio (RTVTR), ridge count and count of white lines [182]. We note that variabilities due to gender cannot be visually deduced from fingerprint images.

3) Gender from Iris: Thomas et al. [251] were the first to predict gender from near-infrared iris images using automated methods. They used a combination of texture features and seven geometric features, including the horizontal, vertical, and Euclidean distances between the pupil center and the iris center, to perform gender classification. Classification was performed by bagging $100 \mathrm{C} 4.5$ decision trees, which resulted in an accuracy of $75 \%$ on a dataset of 28,000 iris-images, and an improved $80 \%$ accuracy when considering only Caucasian subjects.

Furthermore, Lagree and Bowyer [135] continued this line of research by exploring additional texture features (extracted by texture filters such as "spot detectors" and "line detectors"), and without any geometric features. The authors recorded an accuracy of $62 \%$ on a 600 -image dataset of 60 different subjects. The reduced performance, compared to [251], is attributed to the use of a sequential minimal optimization (SMO) support vector classifier, as well as on the difference in the size of the datasets (a 50-fold difference) that resulted in a much smaller training set. Lagree and Bowyer [135] considered both gender and race classification. An interesting observation in their work was that gender classification from iris is a more challenging task than race prediction, and race prediction is harder on females than on males. A related work can be found in [16] where Bansal et al. combined statistical features and texture features using wavelets, and designed a gender prediction model using SVM that achieved an accuracy of $85.68 \%$.

4) Gender from Body: Some biometric traits such as gait require the collection of full body images. Body-based classification of gender has received much attention since the human body has a number of cues for distinguishing gender, as well as the fact that body shape and gait have the potential to reveal the human gender from a longer distance. These cues include 
TABLE III

OVERVIEW OF FACE-BASED GENDER CLASSIFICATION ALGORITHMS. ABBREVIATIONS USED: PRINCIPAL COMPONENT ANALYSIS (PCA), INDEPENDENT COMPONENT ANALYSIS (ICA), SUPPORT VECTOR MACHINES (SVM), GAUSSIAN PROCESS CLASSIFIERS (GPC), ACTIVE APPEARANCE MODEL (AAM), LOCAL BINARY PATTERN (LBP), ACTIVE SHAPE MODEL (ASM), DISCRETE COSINE TRANSFORM (DCT), SEMI-SUPERVISED DISCRIMINANT ANALYSIS (SDA).

\begin{tabular}{|c|c|c|c|c|}
\hline Work & Features & Classifier & $\begin{array}{l}\text { Datasets used } \\
\text { for evaluation }\end{array}$ & $\begin{array}{c}\text { Performance } \\
\text { numbers }\end{array}$ \\
\hline Golomb et al. (1990) [79] & Raw pixels & Neural network & Private, 90 images & $91.9 \%$ \\
\hline Gutta et al. (1998) [92] & Raw pixels & Hybrid classifier & FERET, 3006 images & $96.0 \%$ \\
\hline Sun et al. (2002) [245] & PCA & SVM & Private, 400 images & $91.1 \%$ \\
\hline Moghaddam and Yang (2002) [169] & Raw pixels & SVM & FERET, 1,776 images & $96.6 \%$ \\
\hline Jain and Huang (2004) [108] & ICA & LDA & FERET, 500 images & $99.3 \%$ \\
\hline Khan et al. (2005) [119] & PCA & Neural network & Private, 400 images & $88.7 \%$ \\
\hline Sun et al. (2006) [244] & LBP & Adaboost & FERET, 2,000 images & $95.75 \%$ \\
\hline Kim et al. (2006) [122] & Raw pixels & GPC & AR, 515 images & $97.0 \%$ \\
\hline Saatci and Town (2006) [221] & AAM & SVM & Private, 90 images & $94.8 \%$ \\
\hline Yang and Ai (2007) [281] & LBP & Adaboost & Private, 3,540 images & $96.32 \%$ \\
\hline Bekios-Calfa et al. (2007) [18] & $\begin{array}{l}\text { PCA } \\
\text { LCA }\end{array}$ & $\begin{array}{l}\text { SVM } \\
\text { LDA } \\
\text { LDA }\end{array}$ & $\begin{array}{c}\text { UCN (nonpublic), 10,700 images } \\
\text { FERET, } 994 \text { images } \\
\text { PAL, } 576 \text { images }\end{array}$ & $\begin{array}{l}93.46 \% \pm 1.65 \% \\
93.57 \% \pm 1.39 \% \\
93.57 \% \pm 1.39 \%\end{array}$ \\
\hline Xia et al. (2008) [275] & LBP, Gabor & SVM & CAS-PEAL, 10,784 images & $93.74 \%$ \\
\hline Mäkinen and Raisamo (2008) [157] & LBP & SVM & FERET, 411 images & $86.54 \%$ \\
\hline Baluja and Rowley (2008) [15] & Raw pixels & Adaboost & FERET, 2,409 images & $93 \%$ \\
\hline Gao and Ai (2009) [72] & ASM & Adaboost & Private, 1,300 images & $92.89 \%$ \\
\hline Toews and Arbel (2009) [254] & SIFT & Bayesian & FERET, 994 images & $83.7 \%$ \\
\hline Shan (2010) [234] & LBP & Adaboost & LFW, 7,443 images & $94.44 \%$ \\
\hline Guo et al. (2009) [83] & LBP, HOG, BIF & SVM & YGA, 8,000 images & $89.28 \%$ \\
\hline Wang et al. (2010) [265] & SIFT, context & Adaboost & FERET, 2,409 images & $95.0 \%$ \\
\hline Nazhir et al. (2010) [176] & DCT & KNN & SUMS, 400 images & $99.3 \%$ \\
\hline Ross and Chen (2011) [219] & LBP & SVM & CBSR NIR, 3,200 images & $93.59 \%$ \\
\hline Cao et al. (2011) [22] & Metrology & SVM & MUCT, 276 images & $86.83 \%$ \\
\hline Hu et al. (2011) [105] & Filter banks & SVM & Flickr, 26,700 images & $90.1 \%$ \\
\hline Bekios-Calfa et al. (2011) [19] & SDA & PCA & Multi-PIE, 337 images & $88.04 \%$ \\
\hline Shan (2012) [235] & Boosted LBP & SVM & LFW, 7,443 & $94.81 \%$ \\
\hline Ramón-Balmaseda (2012) [203] & LBP & SVM & MORPH, LFW, Images of Groups, 17,814 & $75.10 \%$ \\
\hline Jia and Cristianini (2015) [113] & Multi-scale LBP & C-Pegasos & Private, 4 million images & $96.86 \%$ \\
\hline
\end{tabular}

body sway, waist-hip ratio, and shoulder-hip ratio (see [164]); for example, females have a distinct waist-to-hip ratio and swing their hips more, whereas males have broader shoulders and swing their shoulders more.

At the same time, extracting gender from body is negatively impacted by several influential factors which - in the case of side profile images - include shoes, background and clothes.

In addition, while the gait cycle contains plenty of information, it also introduces a high feature dimensionality, increasing computational complexity. To reduce dimensionality (a critical step for obtaining better gender estimation accuracy [177]), often, the human silhouette is extracted and a statistical feature - namely an average silhouette - is computed. This is referred to as the gait energy image (GEI). The GEI has been reported to be a good feature for gait and gender recognition because it is robust to silhouette errors and noise (see Figure 5 and also [286], [44]). To again simplify analysis, the body is divided into different regions, such as legs, waist, buttocks, back, chest and head, and these are analyzed separately for gender cues. Results have shown that the head and chest regions contribute significantly to gender cues.

From a computer vision point of view, human gait analysis can be appearance-based or it can be model-based, where characteristics such as height, step-frequency, or angle between two thighs are extracted. Given that extracting such individual characteristics can be a challenging task, most gait recognition algorithms are appearance-based. Table IV gives an overview of body-based gender classification algorithms with the best reported performance being $97.2 \%$ by Shan et al. [236], who fused GEI and facial features. The GEI-based SVM algorithm obtained a classification rate of $93 \%$.

5) Gender from Hand: Extracting gender information from the hand dates back to forensics and archaeological efforts that deduced gender from skeletons with damaged skeletal regions that are traditionally good indicators of gender, viz., the pelvis, skull-region, and larger long bones (for single populations). In the biometrics context, hand-based gender classification is useful as hand images are typically acquired under controlled position, orientation and illumination, resulting in lesser vari- 
TABLE IV

GENDER CLASSIFICATION BASED ON BODY FEATURES. ABBREVIATIONS USED: GAIT ENERGY IMAGE (GEI)(EQUIVALENT TO AVERAGE SILHOUETTE), WEIGHTED AVERAGE SILHOUETTE COMPONENTS (WASS), CORRECT CLASSIFICATION RATE (CCR), CANONICAL CORRELATION ANALYSIS (CCA), Histogram OF ORIENTED GRADIENTS (HOG), MALE (M), FEMALE (F).

\begin{tabular}{|c|c|c|c|c|}
\hline Work & Feature extraction & Classification & Datasets & CCR \\
\hline \hline Cao et al. [23] (2008) & HOG & Adaboost, and Random forest algorithms & MIT pedestrian database (600M, 288F) & $75 \%$ \\
\hline Chen et al. [29] (2009) & 8 WASS & Euclidean distance to WASS-templates & IRIP Gait (32M, 28F) & $93.3 \%$ \\
\hline Li et al. [144] (2008) & 7 WASS & Euclidean distance to WASS-templates & CASIA Gait DB (31M, 31F) & $93.28 \%$ \\
\hline Yu et al. [286] (2009) & GEI & SVM & CASIA Gait DB (31M, 31F) & $95.97 \%$ \\
\hline Yoo et al. [283] (2005) & figure sequential & Human ID (84M, 16F) & $96.7 \%$ \\
\hline Shan et al. [236] (2008) & $\begin{array}{c}\text { CCA-feature fusion of GEI } \\
\text { and face images }\end{array}$ & SVM (RBF) & CASIA Gait DB (31M, 31F) & $97.2 \%$ \\
\hline
\end{tabular}

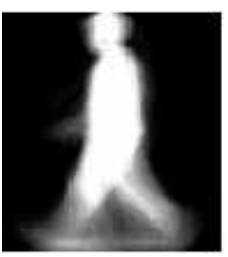

(a)

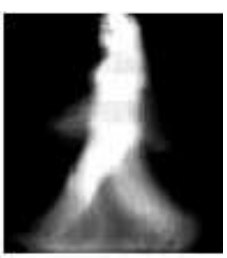

(d)

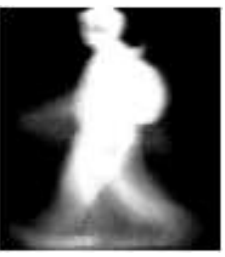

(b)

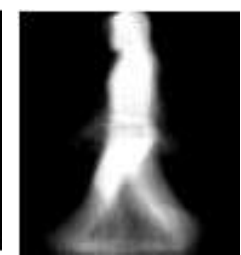

(e)

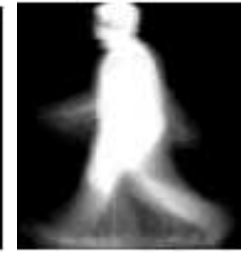

(c)

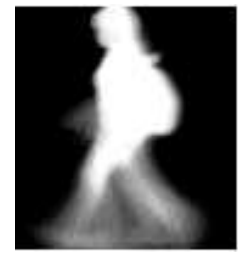

(f)
Fig. 5. Gender from body: A human silhouette is extracted by subtracting the background and, subsequently, an average silhouette is computed, constituting the gait energy image, GEI. The image depicts male ((a)-(c)) and female ((d)(f)) GEIs [44]. Specifically head and chest regions were observed to provide pertinent gender cues.

ability than, for example, facial images which are known to be affected by many factors such as facial expression changes.

In terms of suitability of analyzing hands for gender classification, it has been noted that hand breadth measurements as well as the index to ring finger ratio offer a strong insight on the gender of a person, but at the same time, these can vary between populations and are further influenced by genetics, environment and social conditions. Good studies on this can be found in Kanchan and Krishan [116] and Scheuer and Elkington [231]. Falsetti [58] and Lazenby [140] used measurements of metacarpals of the human hand and obtained gender classification rates of up to $92 \%$. Another approach to determine the gender by Krishan et al. [127], employed the dimensions of hand and feet. Their study employed a test database containing 123 men and 123 women aged 17-20 years old from North India. By analyzing hand length, hand breadth, foot length and foot breadth to classify gender, the study concluded that left foot breadth provided the highest accuracy, which was recorded to be $86.9 \%$.

Interestingly, while most studies on the specific topic are of anthropological and psychological nature, Amayeh et al. [8] adopted a computer-vision approach, in their effort to find gender specific traits in the shape of a hand. They segmented the hand into 6 parts (hand palm and fingers) (see Figure 6), and each part was represented by Zernike moments [121] and Fourier descriptors. Different fusion methods (feature-level, score-level and decision-level) were used to combine these descriptors, and in a very small database of 40 subjects, the method achieved an accuracy of $99 \%$.
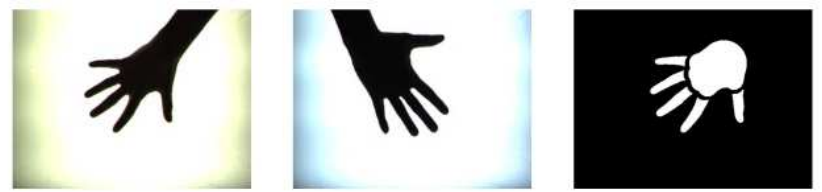

Fig. 6. Hand-based gender classification [8]. A female hand (left), a male hand (middle), and a segmented hand (right).

6) Gender from Speech: In the effort to classify gender, it is only natural to employ speech, since there are substantial perceived differences between typical male and female voices. Towards achieving audio based-automated gender recognition (AGR), speech signals can provide reliable traces, especially in cases of adults, while less reliable traces can be found in infants and elderly individuals (Madry et al. [155]). Audiobased gender traces can be of voice source-character (such as pitch frequency), or can be of vocal tract-character (such as the first four formants with their respective frequency, amplitude and bandwidth [11]). For voice-based approaches, the gender classification problem remains open. This is because, while the $170-275 \mathrm{~Hz}$ female frequency range is disjoint and nicely differentiable from the male equivalent of $112-146 \mathrm{~Hz}$ ([273]), in real world conditions consisting of background noise, low recording quality and variabilities in the physical and emotional state of a subject, human voices can be obfuscated, turning audio-based AGR into a significant challenge [197]. In clean speech data, Childers and Wu [31] analyzed ten vowels to predict the gender. Using a database of 52 talkers $(27$ male and 25 female), the method was recorded to have $100 \%$ gender classification accuracy. Sorokin and Makarov [239] found that features such as the instant of maximum glottis area, the maximum derivative of the area, the slope of the spectrum of the glottal airflow volume velocity pulse, the amplitude ratios of harmonics of this spectrum, and the frequency pitch, can result in an accuracy of $94.7 \%$ for male voice recognition and $95.9 \%$ for female voice recognition. A comparative study of 
To appear in IEEE Transactions on Information Forensics and Security (TIFS), 2015

AGR approaches can be found in the work of Walawalkar et al. [264], where different classifiers such as nearest neighbor classifier and SVMs were evaluated.

7) Hybrid approaches: In addition to the aforementioned purely-image or purely-audio based gender classification approaches, recent work has sought to further the gains of such approaches by combining them with video sources, resulting in audio-visual cues that offer a more comprehensive gender analysis, as well as higher resilience to degradation of any of these sources. Interesting work that employed this approach can be found in Pronobis and Magimai-Doss [196] which explored different audio and visual features, and proceeded to fuse both modalities at the classifier level. Experiments conducted on the BANCA corpus, verified the usefulness of this hybrid approach by showing that the integration of audio-visual cues indeed yielded higher resilience and better performance in noisy conditions. El Shafey et al. [55] employed Total Variability (i-vectors) and Inter-Session Variability (ISV) modeling techniques for audio-visual gender estimation, achieving accuracy rates of up to $98 \%$ on the MOBIO datase $t^{2}$ containing 100 male and 52 female subjects.

Similar to the aforementioned audio-visual approaches, other hybrid approaches seek to combine different biometric modalities in order to increase gender classification performance. Notable related work includes the work of Shan et al. [237] which combines gait and facial features. Specifically, this work employed Adaboost and SVMs to classify human GEIs along with facial features that were related by canonical correlation analysis (CCA) on the CASIA Gait Database (Dataset B) [285] consisting of 124 subjects (93 male, 31 female), and achieved an overall classification rate of up to $99.0 \% \pm 1.3 \%$ for males and $92.0 \% \pm 4.6 \%$ for females.

8) Databases: One distinct advantage of gender classification is that classical databases such as FERET ${ }^{3}$, MORPHII, CAS-PEAL and LFW already contain gender annotated information, while other face-based databases can be easily annotated with gender information by visual examination, as opposed to, for example, age information. Additionally, it is often the case that baseline-performances for gender classification are provided for databases such as FERET.

In Table $\mathrm{V}$ we list different databases that have been used to evaluate gender classification algorithms and can thus serve as test-beds for developing novel gender-classification algorithms.

\section{B. Age estimation}

The ability to estimate age is considered to be an important trait among humans, for evolutionary reasons. Specifically humans learn early on to reliably estimate age of their peers based on holistic face features (e.g. outline of the face, face shape, skin texture), local face features (e.g. areas of the eyes, nose and forehead), as well as related configuration (e.g. symmetry) [212], [172]. From this category of facial features, age can be quantified from features such as the craniofacial index (see Ramanathan and Chellappa [200]), wrinkles, skin

\footnotetext{
${ }^{2} \mathrm{https}: / / \mathrm{www}$. idiap.ch/dataset/mobio

${ }^{3}$ http://www.nist.gov/itl/iad/ig/colorferet.cfm
}

quality [17], facial hair and chin line (Alberta et al. [5]). Age can also be estimated using other body features such as hands, gait, clavicle (collarbone), the pubic bone, and the teeth [174].

A main challenge in estimating age - whether this estimation is performed by humans (human-estimated visually perceived appearance age) or by machines (algorithmically estimated age - comes from the fact that the aforementioned features are a function of many unknowns, including genetics, health condition, living style, working environment, and sociality (Alley [7], Rhodes [212]), all of which vary across people, thus impacting age progression differently from person to person. This variability motivates research in algorithmic age estimation, which - as suggested by a recent study by Han et al. [95] - can often outperform humans at the same task.

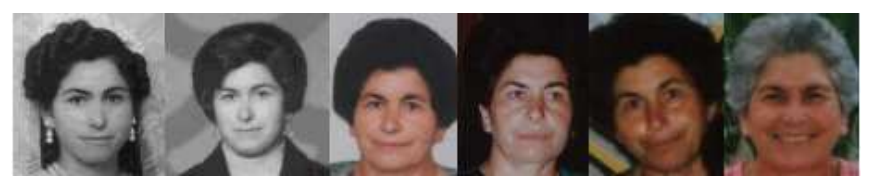

Fig. 7. The human face over a span of time: images obtained from FG-NET. The images (from left to right) represent a subject in FG-NET at the ages of $18,30,35,48,52$ and 61 years old. Facial features that have changed with age include the facial shape, the chin line, and skin quality.

Below, we will describe different methods of automated age estimation, focusing on the challenging module of age image representation. As in the case of gender classification, the task of automated age estimation can be separated into two modular subtasks; the aforementioned part of age image representation during which features are extracted and which will receive most of our focus here, and the modular part of automated classification or regression where the extracted features lead to either a classification decision (corresponding to say a binary decision on whether a person is 'young' or 'old'), or where the extracted features are used in a regression framework to output a more refined decision (corresponding to, for example, an estimate of a person's age). Table VI offers an overview of recent age estimation works and their reported performance. It is here worth mentioning the study by $\mathrm{Fu}$ et al. [68] that focuses on automated analysis of age progression, discussing automated age synthesis and also age estimation techniques.

1) Age from Face:

a) Geometric based approaches: Anthropometric (geometric based) approaches are mainly based on the cranio-facial development theory (see Alley [7]), where - in this case - the growth of the human head is modeled based on a number of anthropometric measurements over a span of time, from infancy to adulthood (see for example the case exhibited in Farkas [60] corresponding to 57 landmarks or fiducial points). Often, what provides more meaningful conclusions on age are ratios between different anthropometric measurements. This ratio-based approach was employed by Ramanathan and Chellapa [200] who used 8 such distance-measure ratios to model age progression. Their model was tested on a database partly adopted from FG-NET ${ }^{4}$, and was shown to provide 8-15\% improvement in face recognition performance, on ages up to

${ }^{4}$ www.fgnet.rsunit.com 
TABLE V

EXAMPLES OF DATABASES THAT HAVE BEEN USED FOR EVALUATING AUTOMATED FACE AND BODY GENDER ESTIMATION METHODS.

\begin{tabular}{|c|c|c|}
\hline Name & Number of Subjects & Acquisition \\
\hline Georgia Tech Database $^{a}$ & 50 & 15 facial images per subject, different pose and lighting conditions \\
\hline GavabDB $^{b}$ & 61 & 427 facial images of 3D meshes, 9 3D-images provided per subject \\
\hline MORPH $^{c}[214] \mathrm{I}$ and II & $515+4000$ & multiple face shots per subject \\
\hline LFW: Labeled Faces in the Wild ${ }^{d}$ & 1680 & 13,233 images acquired under large variability in pose, expression and illumination \\
\hline MIT AI Gait Data ${ }^{\mathrm{e}}$ & 24 & indoor acquisition of 194 gait-sequences \\
\hline CASIA Gait Dataset: (Dataset B) [285] & 124 & multiple sequences of outdoor walking subjects, extracted silhouettes available \\
\hline CMU Mobo Database [81] & 25 & subjects walking on treadmill (slow, fast, inclined and carrying a ball) \\
\hline Gait Challenge Database $^{\mathrm{f}}$ & 122 & 1870 sequences spanning 5 covariates \\
\hline 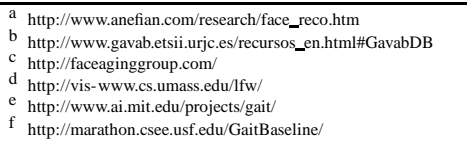 & & \\
\hline
\end{tabular}

18 years. In terms of drawbacks, anthropometric-based models tend to be better for only differentiating between infants and adults. Furthermore, the corresponding measurements and their associated ratios can only be reliably determined from frontal or 3D images. An early 3D approach by Xia et al. [274], which was tested on the FRGCv2 dataset with 466 scans, was shown to achieve a mean absolute error (MAE) of 3.29 years for 4 age classes.

b) Approaches based on appearance models: Another image representation approach relates to appearance models, and considers texture and shape features (and thus indirectly anthropometry and wrinkles), for the purpose of determining the human age. Early approaches were presented by Kwon and Lobo [132], [133], analyzing wrinkles in facial regions such as the forehead and in the periocular region. This analysis determined the classification of young and senior adults. A recent related study by Guo et al. [88] obtained best reported results (MAEs $=2.6$ years, tested on the YGA database). These results were obtained by simultaneously considering age and gender automatic estimation, biologically-inspired features and manifold learning techniques.

Approaches based on active appearance models (AAMs) (see [33]) form a special class under the aforementioned appearance-based models. Interesting work can be found in [139] (Lanitis et al.) which extended the AAMs by proposing an aging function to describe the variation in age. In this aging function, the input is a vector containing 50 raw model parameters learned from the AAMs. The proposed function defines the relationship between the age of the individuals and the parametric description of the face images. Based on agesimulation (using a 'Weighed Person Specific Age Simulation' method), the age in all images was normalized to eliminate the effects of aging. This approach led to an improvement of face recognition performance from $63 \%$ to $71 \%$ and from $51 \%$ to $66 \%$, on two different datasets. Other interesting AAM-based approaches have been employed by Guo et al. [84] and by Lakshmiprabha [136].

c) Approaches based on aging pattern subspaces: Aging pattern subspaces are formed from a sequence of images per person representing the chronological progress of aging, i.e., representing an aging pattern. Specifically, each point in the subspace corresponds to one aging pattern. The age of an unseen face image is determined by the projection in the subspace that can best reconstruct the face image. The position of the face in the subspace indicates the age of the associated face. In this approach, which was introduced by Geng et al. [74], [75], this sequence of images forms what is specifically known as the AGing pattErn Subspace (AGES), which is then properly represented - for each person, at each given age - by AAMs (missing ages are synthesized using an EM-like iteration algorithm). In the testing phase, an image of a face is matched to the faces of the subspace (i.e., of AGES), and the degree of the matching is then translated into an estimate of the age. This approach was tested [74], [75] on the FG-NET face database, resulting in a reported MAE of 6.77 years. While promising, this approach has the drawback that it requires multiple images per subject in order to learn age progression. Additionally, wrinkles are - in general - not sufficiently represented by AAMs, and are thus downplayed in the process of estimation. A related approach can be found in the recent work by Guo et al. [88], [89] which draws from the feed-forward path theory of the cortex for visual processing. In this approach, after sequentially filtering an image with a Gabor filter and a standard deviation based filter, the obtained features are subjected to PCA resulting in a lower-dimensional bio-inspired-features (BIF). Recently, deeplearning aging pattern approaches were presented by Wang et al. [267] and Tian and Chen [253].

d) Approaches based on age manifolds: First presented by $\mathrm{Fu}$ et al. [71], the main idea behind this approach is that functions that estimate age can be of reduced dimensionality, i.e., will take as input only a reduced number of characteristic parameters. Such functions are used in conjunction with manifold learning algorithms which reveal these characteristic parameters and find a low-dimensional representation of the data (cf. Seung and Lee [232]). The resulting manifold can be linear (Yan et al. [280]) or non-linear (Tenenbaum et al. [250]). Furthermore Guo et al. [84] explored learning manifolds based on projections that preserve orthogonal locality. This approach was reported to provide an MAE of 5.3 years on FG-NET, if the size of the training data is large enough. The related linear manifold embedding method is referred to as orthogonal locality preserving projections (OLPP). Enhanced age estimation performance was recorded by $\mathrm{Li}$ et al. [143] who performed age-manifold learning under locality preserving requirements as well as under ordinal requirements. 
e) Automated age classification or regression: As previously mentioned, following the feature extraction in the age-image-representation part, one can proceed with age estimation in the form of automated classification or automated regression. A useful exposition of classification and regression approaches can be found in Guo et al. [85] which places emphasis on techniques based on support vector regression (SVR) and support vector machines (SVM). In brief, the authors conclude that comparable results can be obtained using both approaches. Furthermore, the work of Guo [82] discusses the application of SVMs in learning an estimator or recognizer for the purpose of age extraction. Finally, it is worth noting that classification and regression approaches can indeed be combined to form hybrid approaches (cf. Guo et al. [86]).

2) Age from Body: In the context of age estimation, Lu and Tan [150] recently analyzed gait. In this setting - where, as the authors state, gait appearances vary between males and females even within the same age group - the approach was based on learning a multilabel-guided subspace to better characterize and correlate the age and gender information of a person for estimating human age. The authors extract Gabor features including both Gabor magnitude and Gabor phase information of a gait sequence, and perform multiple feature fusion to enhance the age estimation performance. The related experimental results present an MAE of 3.02 years on the USF database [225] consisting of 1870 gait sequences from 122 subjects ( 85 male and 37 female subjects of age between 19 and 59 years old).

3) Age from Hand: One characteristic work in this setting is that by Shamir [233] which presented a new approach of automatic age classification based on the features that appear on the back of the human hand. Estimating age from the hand, enjoys - as opposed to age estimation from the face - the advantage of being privacy preserving, and the advantage of being invariant to facial makeup and plastic surgery. Experimental results in the same work show that the method can differentiate between older and younger individuals with an accuracy of $88 \%$. The hand photos were taken from Baltimore Longitudinal Study of Aging $^{5}$ (BLSA). The dataset includes 106 subjects, and 212 images (two from each subject).

4) Databases: An increasing number of databases feature biometric data related to different ages. We report some of these in Table VII.

\section{Ethnicity and race classification}

The traditional definition of race is related to biological factors and often refers to a person's physical appearance corresponding to traits such as skin color, eye color, hair color, bone/jaw structure, face and body shape, and other traits, while the traditional definition of ethnicity is more related to sociological factors and it relates primarily to cultural identifiers such as nationality, culture, ancestry, language as well as beliefs. The terminology overlaps and is often used interchangeably in the biometric literature, and hence we will also adopt the same loose convention here.

\footnotetext{
${ }^{5}$ http://www.blsa.nih.gov/
}

a) Limitations and challenges in race classification: Before addressing some of the progress in automated race classification, it is worth touching upon some points which suggest that ethnicity- or race-categorization is a rather challenging problem. The first important point - as indicated in the book of Mordini and Tzovaras [170] - is that intra-class variation can often be significantly larger than the inter-class variation between races. Similarly, recent findings by geneticists (cf. [161], [9]) show that there is often no clear relation between races on one hand and the frequency of particular genetic variants on the other. In the same vein, Rosenberg et al. [218] reported that within-population differences among individuals account for $93 \%-95 \%$ of genetic variations, whereas major ethnic groups differentiate only by $3 \%-5 \%$. At the same time, however, the work in [218] identified six main genetic clusters, five of which corresponded to major geographic regions, and sub-clusters that often corresponded to individual populations.

Related to the challenging nature of race-categorization is the "other race effect", where humans charged with a face recognition task are observed to perform significantly better when having to recognize the face of a person from their own race (cf. O'Toole et al. [184]). Interestingly, such an effect has also been witnessed in automated face recognition [192][184]. Specifically, this "other race effect" was observed in an international face recognition competition where a fusion of algorithms from Western countries performed better in recognizing Caucasian faces, while a fusion of algorithms from East Asian countries performed better in recognizing East Asian faces.

1) Race from Face: Before presenting some research highlights on this topic, we note that as with gender and age estimation, race estimation typically comprises of a feature representation module, where the features are extracted, and a subsequent classification module, where the extracted features are categorized into a discrete number of race classes. As before, approaches for classification include geometric based approaches [60], holistic/appearance-based approaches (involving color, texture and shape-based algorithms), as well as approaches based on local feature descriptors. While it is generally agreed that humans perform race classification by employing a feature based approach [271], many of the promising computer vision solutions that we will see later on employ approaches based on holistic and local features.

We describe some of these approaches below. Table VIII summarizes these approaches. The interested reader can also explore a recent survey by $\mathrm{Fu}$ et al. [65] which provides a comprehensive study on human and automated analysis of race classification.

a) Approaches based on chromaticity: Chromaticity, or skin tone, has long been employed as the primary feature for race classification; see for example Xie et al. [278], Roomi et al. [217], and Tariq et al. [249]. The latter work used human silhouette images, and achieved classification rates of $80.37 \%$ for 'East and Southeast Asian' subjects, $78.67 \%$ for 'White Caucasian' subjects, $58.21 \%$ for 'Black' subjects, and $33.33 \%$ for 'South Asian' subjects. We note here that chromaticitybased race classification has the limitation of being strongly sensitive to illumination changes. 
TABLE VI

AgE ESTIMATION TECHNOLOGIES BASEd ON CLASSIFICATION. ABbREVIATIONS USED: ACTIVE APPEARANCE MODEL (AAM), APPEARANCE MODEL

(APM), AGE MANIFOLD (AMF), BIO INSPIRED FEATURES (BIF), DISTANCE METRIC LEARNING (DML), CUMULATIVE ATTRIBUTES (CA),

CONVOLUTIONAL NEURAL NETWORK (CNN), DEEP LEARNED AGING PATTERN (DLA), GRAPH-BASED COMPOSITIONAL AND DYNAMIC MODEL (GB), ORDINAL DiSCRIMINATIVE AGING (PLO), MALE (M), FEMALE (F).

\begin{tabular}{|c|c|c|c|c|c|}
\hline Work & Features & $\begin{array}{l}\text { Datasets used } \\
\text { for evaluation }\end{array}$ & $\begin{array}{c}\text { Number of } \\
\text { subjects }\end{array}$ & Accuracy & $\begin{array}{c}\text { Classification } \\
\text { Regression }\end{array}$ \\
\hline Guo et al. (2008) [84] & $\begin{array}{c}\text { AAM } \\
\text { AMF (OLPP) }\end{array}$ & $\begin{array}{c}\text { FG-NET } \\
\text { YGA }\end{array}$ & $\begin{array}{c}82 \\
800(\mathrm{M}) 800(\mathrm{~F})\end{array}$ & $\begin{array}{c}88 \% \\
83 \%(\mathrm{M}), 82 \%(\mathrm{~F})\end{array}$ & $\begin{array}{l}\mathrm{C} \\
\mathrm{C}\end{array}$ \\
\hline Kwon and Lobo (1999) [133] & $\begin{array}{l}\text { facial ratios, } \\
\text { wrinkle index }\end{array}$ & Private & 47 & $100 \%$ & $\mathrm{C}$ \\
\hline Kanno et al. (2001) [117] & APM & Private & $110(\mathrm{M})$ & $80 \%$ & $\mathrm{C}$ \\
\hline Lanitis et al. (2004) [138] & AAM & Private & 40 & MAE $=3.82-5.58$ & $\mathrm{R}$ \\
\hline Zhou et al. (2005) [296] & APM & FG-NET & 82 & MAE $=5.81$ & $\mathrm{R}$ \\
\hline Ueki et al. (2006) [256] & APM (raw image) & WIT-DB & $3000(\mathrm{M}), 2500(\mathrm{~F})$ & $50 \%(\mathrm{M}), 43 \%(\mathrm{~F})$ & $\mathrm{C}$ \\
\hline Takimoto et al. (2006) [248] & APM & HOIP & 113(M), 139(F) & $57.3 \%, 54.7 \%$ & $\mathrm{C}$ \\
\hline Takimoto et al. (2007) [247] & ANN & HOIP & 113(M), 139(F) & $3.0(\mathrm{M}), 4.4(\mathrm{~F})$ & $\mathrm{R}$ \\
\hline Geng et al. (2007) [74] & AGES & MORPH & 515 & $\approx 70 \%$ & $\mathrm{C}$ \\
\hline Fu and Huang (2008) [69] & AMF (OLPP) & YGA & $800(\mathrm{M}), 800(\mathrm{~F})$ & $\mathrm{MAE}=8.0(\mathrm{M}), 7.8(\mathrm{~F})$ & $\mathrm{R}$ \\
\hline Zhuang et al. (2008) [298] & APM (patches) & YGA & $800(\mathrm{M}), 800(\mathrm{~F})$ & MAE $=5.4(\mathrm{M}), 6.33(\mathrm{~F})$ & $\mathrm{R}$ \\
\hline Guo et al. (2008) [85] & quadr. regression & YGA & 4000(M), 4000(F) & $\mathrm{MAE}=6.0(\mathrm{M}), 5.5(\mathrm{~F})$ & $\mathrm{R}$ \\
\hline Ni et al. (2009) [178] & APM (patches) & Web data, FG-NET & 78711 images & MAE $=7.42$ & $\mathrm{R}$ \\
\hline Guo et al. (2009) [88] & $\mathrm{APM}(\mathrm{BIF})+\mathrm{AMF}$ & YGA & $800(\mathrm{M}), 800(\mathrm{~F})$ & $89.7 \%$ & $\mathrm{C}$ \\
\hline Guo et al. (2009) [89] & APM (BIF) & YGA & $800(\mathrm{M}), 800(\mathrm{~F})$ & $88 \%(\mathrm{M}), 85 \%(\mathrm{~F})$ & $\mathrm{C}$ \\
\hline Xiao et al. (2009) [276] & $\overline{\text { DML }}$ & FG-NET & 82 & $84 \%$ & $\mathrm{C}$ \\
\hline Yan et al. (2009) [279] & SSE & FG-NET & 82 & MAE $=5.21$ & $\mathrm{R}$ \\
\hline Ni et al. (2009) [178] & RMIR & Web data, MORPH & $77,021+55,608$ & $\mathrm{MAE}=8.6$ & $\mathrm{R}$ \\
\hline Suo et al. (2010) [246] & GB & FG-NET & 82 & $82.7 \%$ & $\mathrm{C}$ \\
\hline Lakshmiprabha et al. (2011) [136] & AAM & FG-NET & 82 & $77 \%$ & $\mathrm{C}$ \\
\hline Li et al. (2012) [143] & PLO & FG-NET & 82 & $88 \%$ & $\mathrm{C}$ \\
\hline Chen et al. (2013) [28] & CA - AAM & $\begin{array}{l}\text { FG-NET } \\
\text { MORPH }\end{array}$ & $\begin{array}{c}82 \\
515\end{array}$ & $\begin{array}{l}\mathrm{MAE}=4.67 \\
\mathrm{MAE}=5.88\end{array}$ & $\begin{array}{l}\mathrm{R} \\
\mathrm{R}\end{array}$ \\
\hline Wang et al. (2015) [267] & CNN - DLA & $\begin{array}{l}\text { MORPH } \\
\text { FG-NET }\end{array}$ & $\begin{array}{c}515 \\
82\end{array}$ & $\begin{array}{l}\mathrm{MAE}=4.77 \\
\mathrm{MAE}=4.26\end{array}$ & $\begin{array}{l}\mathrm{R} \\
\mathrm{R}\end{array}$ \\
\hline
\end{tabular}

TABLE VII

EXAMPLES OF DATABASES THAT HAVE BEEN USED FOR EVALUATING AUTOMATED AGE ESTIMATION METHODS.

\begin{tabular}{|c|c|c|}
\hline Name & Number of Subjects & Acquisition \\
\hline$\overline{\text { FG-NET }^{\mathrm{g}}}$ & $\overline{82}$ & $\begin{array}{l}1002 \text { color and gray scale images with variation in pose, illumination and expression. Age: } \\
0-69 \text { years. }\end{array}$ \\
\hline MORPH I and II [214] $]^{\mathrm{h}}$ & $515+4,000$ & $\begin{array}{l}\text { Database annotated for age, gender, ethnicity, height, weight and ancestry. Album 1: } 1,724 \\
\text { images corresponding to } 515 \text { subjects, captured between } 1962 \text { and } 1998 \text {. Album } 2: \text { more } \\
\text { than } 20,000 \text { images from more than } 4,000 \text { subjects. Age: } 27-68 \text { years. }\end{array}$ \\
\hline WIT-DB [256] & 5,500 Japanese subjects & 2,500 females and 3,000 males $(12,008+14,214$ images respectively). Age: $3-85$ years. \\
\hline HOIP $^{i}$ & 300 & $\begin{array}{l}306,600 \text { images (half males and half females). Age: annotated in } 10 \text { age categories from } \\
15 \text { to } 64 \text { with a range of five years per category, all with neutral expressions. }\end{array}$ \\
\hline 3D Morphable Database [230] & 200 adult +238 teenager & 3D scans (half males and half females) \\
\hline
\end{tabular}

b) Approaches based on global features: Global feature representation, or holistic representation, has been found to preserve configural face information (interrelations between facial regions), which is helpful towards race classification. Classical methods include the work of O'Toole et al. based on PCA of raw pixel intensities [175], [192], achieving accuracies of about $80 \%$ for distinguishing between Japanese and Cau- casian subjects. The work by Lu and Jain [152] employed an LDA-based algorithm to classify 263 subjects into 2 categories (Asian and Non Asian) obtaining a success rate of about $96 \%$.

c) Approaches based on local feature descriptor representation: These include methods proposed by Lin et al. [147], Klare and Jain [124], Heo and Savvides [102], and Hosoi et al. [104]. The latter employed Gabor Wavelet Transformation and retina sampling, along with a SVM classifier. On a 
database containing 771 Asian, 774 European and 446 African subjects, an accuracy of about $94 \%$ was obtained. Zang and Yi [281] examined 11680 Asian and 1016 non-Asian subjects, and employed LBP in an AdaBoost classification to separately determine their ethnicity, gender, and age. For ethnicity classification, the resulting EER was $2.98 \%$. Another approach was presented by Fu et al. [66] who used topographic independent component analysis (TICA) to form a hierarchical multi-level cortex-like mechanism, which achieved a classification rate of $82.5 \%$

Finally, one can also encounter early hybrid approaches that aim to combine local and global features. One such hybrid approach was presented by Ding et al. [50] that boosted local texture and global shape features, resulting in accuracies of up to $98.3 \%$.

2) Race from Iris: Recent research has explored the possibility of deducing race from iris images (see Figure 8). For example, Qiu et al. [198] showed that geometric characteristics of the iris exhibit significant differences across races. This has been further confirmed in [199], [135], [294]. Building on this property, Qiu et al. [198] developed a model to predict ethnicity from iris texture. Their work considered a binary classification problem (Asian vs. non Asian), and employed the CASIA database with 2400 images for the Asian subjects, while for the non Asian subjects it employed the UPOL database $^{6}$ with 384 images of 64 subjects and the UBIRIS database [195] with 1198 images of 240 subjects. Using AdaBoost, the work recorded an accuracy of $85.95 \%$. The images in the UPOL and UBIRIS databases were acquired using visible light, while the images in CASIA were acquired in near-infrared illumination. The authors conducted an experiment to establish whether illumination affects the proposed method for ethnicity classification. The experiment consisted of selecting irises of Asian subjects from UPOL, UBIRIS and CASIA, and attempting to classify images into those captured under visible light versus those captured in the presence of near-infrared illumination. The related results showed a nearrandom classification (of illumination), suggesting that the presented ethnicity classification is due to inherent characteristics of iris texture, rather than illumination. An extension of this work by the same authors [199] computed texture features using 40 Gabor filters formed by eight orientations and five scales. The work used 200 images of Asian subjects from the CASIA database, and 200 images from the BioSecure ${ }^{7}$ database for the non-Asian subjects. The test dataset contained 2,400 additional images of 60 subjects ( 30 from the CASIA and 30 from the BioSecure database). A K-means algorithm was then used to form 64 clusters representing textons that are commonly occurring fundamental texture elements. An image was then analyzed in terms of 64-texton histograms, where each pixel was classified into one of 64 textons, so that an image is represented by a 64-element feature vector. With the above in place, an SVM classifier was then applied, achieving a correct classification rate of up to $88.3 \%$. Lagree and Bowyer [135] focused on analyzing different features

\footnotetext{
${ }^{6} \mathrm{http}: / /$ phoenix.inf.upol.cz/iris/

${ }^{7}$ http://biosecure.it-sudparis.eu/AB/
}

and texture filters. Using SMO support vector algorithms with Weka's default parameter settings ${ }^{8}$, the work reported an accuracy of $90.58 \%$ (the employed dataset consisted of 60 subjects and 600 images).

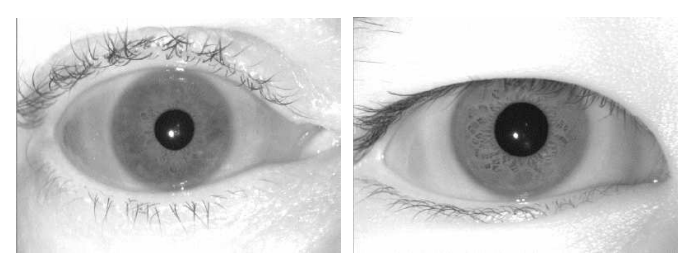

(a)

(b)

Fig. 8. Iris images corresponding to different ethnic groups, (a) a subject with Caucasian ethnicity, (b) a subject with Asian ethnicity [135].

3) Databases: In terms of classical databases - such as the FERET database - the advantage of having annotated ethnicity/race information is diminished by the fact that this information is often statistically unbalanced, simply because such databases were not created with race-classification in mind. This often forced researchers in multi-race classification to combine different databases to ensure a racially balanced and diverse information set. As a consequence of this, one faces the problem of employing different datasets that were created under different acquisition conditions. As a result, recent efforts have been made to create racially diverse databases. We list some of these in Table IX.

\section{Relation between age, gender and race}

It is often the case that a single facial feature can carry information about different soft biometric traits. That is why traits such as age, gender and ethnicity are often treated and categorized simultaneously (cf. [147], [87], [159], [94]) - an approach that is in line with the perceived correlation of these soft biometric traits. In addition to the correlation, exploring this intertwined nature of traits can carry substantial advantages; for example, from a genetic point of view, understanding the interaction of race with aging allows for conclusions on race-based differences in longevity and agingassociated diseases, as well as the possible role of genetic factors in such differences [73].

This perceived correlation between these traits has motivated additional work such as that in [59], which investigated if ethnicity-based gender classification can improve the accuracy of three gender estimation algorithms, namely pixel-based, HOG and LBP. The work concluded that joint treatment is not beneficial and that gender and ethnicity can be estimated separately, due to the fact that features used to estimate gender are shared by all ethnic groups and features used for ethnicity classification are present in both female and male faces.

\section{ANTHROPOMETRIC ATTRIBUtes}

We next focus on soft biometric traits that have been historically used to quantify the geometry and shape of the face, body and skeleton (i.e., of classical anthropometric measures).

\footnotetext{
${ }^{8}$ http://www.cs.waikato.ac.nz/ml/weka
} 
TABLE VIII

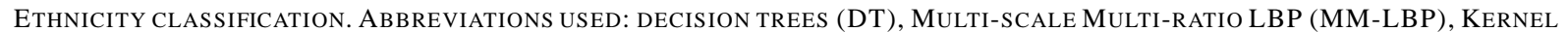
Class-dependent Feature Analysis (KCFA), Oriented Gradient Maps (OGMs), Gate Energy Image (GEI), Support Vector Machines (SVM), CATEGORIES (CAT).

\begin{tabular}{|c|c|c|c|c|c|c|}
\hline Work & Features & Classifier & $\begin{array}{l}\text { Datasets used } \\
\text { for evaluation }\end{array}$ & $\begin{array}{l}\text { Nr. of images / } \\
\text { subjects }\end{array}$ & Cat. & Performance \\
\hline Gutta et al. (98) [92] & RBF networks & inductive DT & FERET & 1009 subj. & 4 & $92.0 \%$ \\
\hline Viola et al. (02) [262] & Haar-features & Adaboost & Private & 4132 images & 2 & Total error $>20.8 \%$ \\
\hline Hosoi et al. (04) [104] & $\begin{array}{l}\text { Gabor Wavelets, } \\
\text { retina sampling }\end{array}$ & SVM & Private & 1991 images & 3 & $94 \%$ \\
\hline Lu and Jain (04) [152] & $\begin{array}{l}\text { LDA ensembles at } \\
\text { multiple scales }\end{array}$ & LDA-based classifier & Private & 263 subj. & 2 & $96.3 \%$ \\
\hline Lu et al. (06) [151] & $\begin{array}{l}\text { Sampled range and } \\
\text { intensity images }\end{array}$ & SVM & Private & $\begin{array}{l}1240 \text { images, } \\
376 \text { subj. }\end{array}$ & 2 & $\begin{array}{c}\text { Average error } \\
\text { rates } 2 \%\end{array}$ \\
\hline Yang and $\mathrm{Ai}(07)$ [281] & $\begin{array}{l}\text { LBP, Chi square } \\
\text { distance }\end{array}$ & AdaBoost & $\begin{array}{l}\text { Private and } \\
\text { FERET }\end{array}$ & 12696 images & 2 & $97 \%$ \\
\hline Tariq et al. (09) [249] & $\begin{array}{c}\text { Shape context and } \\
\text { shape distance } \\
\text { (silhouetted face profiles) }\end{array}$ & KNN & $\begin{array}{l}\text { generated from 3D } \\
\text { face models }\end{array}$ & 441 images & 4 & $33.33 \%-80.37 \%$ \\
\hline Zhang and Wang (09) [293] & 2D and 3D MM-LBP & Adaboost & FRGC v2.0 & 180 subj. & 2 & $99.5 \%$ \\
\hline Zhang et al. (12) [291] & $\begin{array}{l}\text { Uniform LBP } \\
\text { face and gait }\end{array}$ & SVM & Private & 36 subj. & 2 & $93 \%$ \\
\hline Xie et al. (12) [278] & $\begin{array}{l}\text { KCFA + color } \\
\text { based features }\end{array}$ & KNN, SVM & $\begin{array}{l}\text { Private and } \\
\text { MBGC }\end{array}$ & 104,000 images & 3 & $95 \%-98 \%$ \\
\hline Zhang et al. (12)[290] & $\begin{array}{l}\text { GEI: gait / } \\
\text { Gabor: face }\end{array}$ & SVM / Adaboost & Private & 22 subj. & 2 & $>95 \%$ \\
\hline Ding et al. (13) [50] & OGMs in 3D images & Adaboost & FRGC v2.0 & 466 subj. & 2 & $98 \%$ \\
\hline
\end{tabular}

TABLE IX

EXAMPLES OF DATABASES THAT HAVE BEEN USED FOR EVALUATING AUTOMATED RACE OR ETHNICITY CLASSIFICATION METHODS.

\begin{tabular}{|c|c|c|c|}
\hline Name & Number of Subjects & Number of Classes & Ethnicity Distribution \\
\hline FRGC $2.0^{\mathrm{j}}$ & 4007 & 5 & Validation set: $22 \%$ Asian, $68 \%$ White, $10 \%$ Other \\
\hline Color FERET ${ }^{\mathrm{k}}$ & 2,946 & 4 & 1,902 Caucasian, 352 Asian, 464 Oriental, 228 African [92] \\
\hline Cohn-Kaade $^{\mathrm{l}}$ & 210 & 3 & 170 Euro-American, 27 Afro-American, 13 others [153] \\
\hline JACFEE $^{\mathrm{m}}$ & 56 & 2 & 28 Caucasian, 28 Japanese $^{\text {n }}$ \\
\hline EGA [215] & 469 & 5 & 53 African-Americans, 111 Asians, 162 Caucasians, 75 Indian, 68 Latino $^{\circ}$ \\
\hline MORPH-II [214] ${ }^{\mathrm{p}}$ & 78,207 & 3 & 15,996 White, 58,326 Black, 3,885 Other [96] \\
\hline PCSO [96] & 100,012 & 3 & 69,116 White, 26,457 Black, 4,439 Other [96] \\
\hline LFW $^{\mathrm{q}}$ & 4,211 & 3 & 3,501 White, 352 Black, 358 Other [96] \\
\hline \multicolumn{4}{|c|}{ 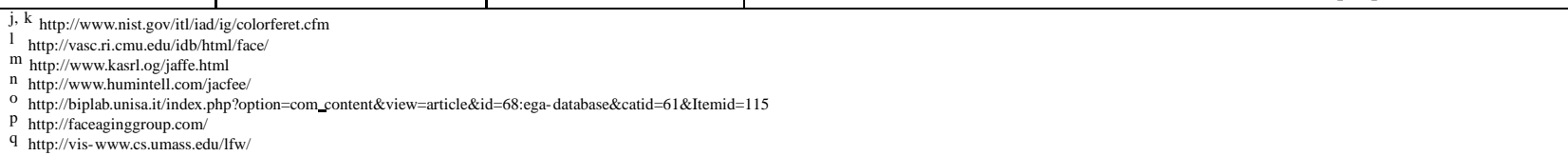 } \\
\hline
\end{tabular}

These traits have become pivotal in the context of geometry extraction - i.e., the localization of facial landmarks related to eyes, mouth, nose, chin, etc. - which is a key step in a number of applications ranging from human identification to gender, ethnicity, and age estimation to emotion and expression recognition [288].

While a number of geometric estimation methods have been developed, these methods have to account for several different factors. Firstly, some of the geometric measurements can be correlated (Farkas [60]); thus, the model used for assessing correlation can impact the accuracy of estimation. Secondly, the estimation methods have to account for variations in sensor and calibration; this is necessary to ensure that the estimated
3D affine measurements are consistent across different systems [35][295]. Thirdly, if there are multiple cameras focusing on the same scene, then it may be necessary to judiciously combine the complementary information provided by these cameras during the estimation process.

"Forensic anthropometry" [126] studies somatometry (measurements of the body) [206], [191], [99], cephalometry (measurements of the head) [107], craniometry (measurements of the skull) [49] and osteometry (measurements of the bones of the skeleton) [242], and is used for person identification in the case of unknown human remains [10]. 


\section{A. Anthropometric features from face}

A number of efforts have been directed to suitably capture and represent different geometric anthropometric measures of the face. As mentioned earlier, the main reason that such facial geometric anthropometric measures are of importance, has to do with the fact that localization of facial landmarks (related to eyes, mouth, nose, chin), is often a key step towards precise geometry-extraction, which is in turn crucial for human identification and a class of other recognition systems [288] that generally employ these traits as trackers. In this context, algorithms such as AAM, active shape model (ASM), and deformable shape model have been used (cf. Ding and Martinez [51]) to obtain such facial landmarks.

Further motivation comes from the fact that - as noted by Nixon in [179] - a judicious combination of these facial features can result in a relatively high degree of distinctiveness for person recognition. In addition, face-based geometric human metrology is an important tool in forensic anthropology [116].

Another line of interesting work is the design of algorithms that robustly recognize people in the presence of occluded and disguised faces. In this setting, Ramanathan and Wechsler [202] combine appearance based approaches (PCA + LDA), and anthropometric / geometric measurements (19 manually extracted geometric measurements of the head and shoulders) via decision-level fusion (neural networks) and feature-level fusion (AdaBoost), to design an algorithm that is robust to occluded and disguised faces. Related work can also be found in Ghalleb et al. [76] where facial measurements ultimately used for human recognition - are extracted based on geometry in the detected face (SMQT and split up SNOW classifier). This resulted in a soft biometric system, which was fused with different classical biometric systems, to achieve an improved matching accuracy.

\section{B. Anthropometric features from the body}

Among the many geometric traits, body height is the most prominent. In extracting this measure, different challenges remain, including that of the human pose which can serve as a primary biasing factor. Therefore, BenAbdelkader and Davis [20] resorted to averaging different body measurements over different poses in order to reduce the pose bias. Other methods that result in robust height estimation can be found in the work by Madden and Piccardi [154] where a person's height was estimated from surveillance video, thus allowing for session-based biometric matching using two disjoint camera views.

Body height can often be estimated together with other geometric measures. For example, the aforementioned work by BenAbdelkader and Davis [20] considered shoulder breadth (bideltoid breadth) in addition to body height, and the two measures were jointly averaged for the purpose of improving multi-target tracking across multiple cameras. In a similar spirit, but in the setting of person identification, Johnson and Bobick [115] extracted four static parameters (body height, torso length, leg length, and step length), achieving a typical error of about $30 \mathrm{~cm}$ for anthropometric measure estimation. Furthermore, BenAbdelkader et al. [32] used height and stride (extracted from gait videos) for person recognition, achieving true recognition rates of $47 \%$ for fronto-parallel sequences of 41 people, and $65 \%$ for non-fronto-parallel sequences of 17 people.

Recently, body shape has been estimated in millimeter wave images (acquired at $94 \mathrm{GHz}$ ) [80]. Contour coordinates and dynamic time warping were used resulting in an EER of $1.33 \%$ on a dataset of 50 individuals (BIOGIGA [171]).

a) 3D techniques in geometric anthropometric measurements: Recently, 3D techniques have been used to obtain geometric anthropometric measurements. Some of these works include the study by Carnicky and Chorvat [24] that focused on the acquisition of 3D measurements with structured light illumination, and the work by Allen et al. [6] that developed a method for fitting high-resolution template meshes to 250 detailed human body range scans from the 3D CAESAR database $^{9}$ with sparse 3D markers to model the variation of different bodies.

Additionally, in the work of Adjeroh et al. [2] spanning face and body metrology, the 1D-measurements in the 3D CAESAR database were used to show a high degree of correlation between certain face and body measurements. Utilizing the correlation structure between measurements, the researchers showed that gender could be estimated with a success rate of $100 \%$ (this corresponded to 44 measurements), and body weight could be estimated with a success rate of $92.5 \%$. Based on the idea that some anthropometric measurements are highly correlated and that missing values can thus be predicted, an iterated closest point (ICP) registration-model and Laplacian mesh was presented by Hasler et al. [98], where a detailed 3D human body shape in the presence of clothes was modeled based on a space of human shapes, learned from a large database of registered body scans. Similarly, by using the visual hull, a method for estimating detailed 3D body shape under clothes was presented by Balan and Black [14]. This work used the HumanEva ${ }^{10}$ dataset. Furthermore, Criminisi et al. [35] computed 3D affine measurements from single perspective views, where affine scene structure could be determined from the image, without knowledge of the camera's internal calibration (e.g. focal length), nor of the explicit human pose. Interesting work can also be found in Godil and Ressler [78] who performed similarity-based retrieval in the 3D CAESAR anthropometric database (3D scans), where the technique was based on body and head shape representation. Finally geometric/anthropometric features have also been exploited to enhance gait recognition accuracy (cf. Moustakas et al. [173]).

\section{Databases}

While some datasets include the annotation of pertinent facial landmarks such as eyes, nose and mouth, these are mainly used for image alignment. Often, more detailed landmark annotation in common datasets is performed manually, in order to capture the necessary measurement ratios, which are often more valuable than the measurements themselves.

\footnotetext{
${ }^{9} \mathrm{http} / / /$ www.hec.afrl.af.mil/cardlab/CAESAR/index.html

${ }^{10} \mathrm{http}: / /$ vision.cs.brown.edu/humaneva/
} 
Notable exceptions include the two paid-for datasets ${ }^{11}$ of the Civilian American and European Surface Anthropometry Resource Project (CAESAR).

CAESAR 3D Anthropometric Database (North American Edition) includes 40 anthropometric / geometric measurements per person, from a North American population sample of 2,400 male and female subjects, aged 18-65. It also includes demographic information and 3D model scans. There are several poses of each person.

CAESAR 3D Anthropometric Database (European Edition) was recorded in the same project as the North American Edition and also features 40 anthropometric / geometric measurements from a European population sample of 2,000 European male and female subjects, aged 18-65. Demographic information as well as 3D scans are provided under several poses.

\section{Medical attributes}

\section{A. Image-based automated self diagnostic methods}

is a novel and promising approach of enhancing classical medical care. Such methods, when applied properly, can provide a broad range of medical benefits, including early detection and prevention of illnesses, as well as efficient treatments when possible. Such methods are needed now more than ever, due to the ever increasing burden on traditional medical care systems. With this need in place, we are already seeing different medical sectors employ computer vision schemes, such as in the crucial area of monitoring the health of elderly people to improve their safety, autonomy and quality of life [216], [186], [266]. Such promising applications, and many others, have introduced the need for algorithms and systems that can reliably monitor the state of different human traits, which in turn accentuates the importance of being able to properly characterize soft biometric traits such as body weight, body mass index $\left(\mathrm{BMI}=\frac{\text { body weight }}{(\text { body height })^{2}}\right)$, or even skin color and quality.

\section{B. Health and weight}

Again in the context of health, body weight and BMI have received increased attention in recent years. This boost in attention is related to health concerns that have been expressed in several countries. For example, it has been stated that more than two-thirds of adults in the United States are now overweight or obese [282], and obesity - which is now recognized as a disease by the American Medical Association ${ }^{12}$ - accounts for nearly one out of every 10 American deaths. Specifically, an increased body weight and thus BMI ${ }^{13}$, is often associated with many health problems such as diabetes, heart disease, certain cancers, strokes, osteoarthritis and gallstones, all of which cost the United States 223 billion per year ${ }^{14}$. This has motivated the use of automated techniques for estimating body

\footnotetext{
${ }^{11}$ http://store.sae.org/caesar/\#3dna

${ }^{12}$ www.ama-assn.org/go/obesity

${ }^{13}$ The use of BMI as a sole measure of obesity has been challenged in the medical literature.

${ }^{14} \mathrm{http} / / / \mathrm{www} . h e a l t h . h a r v a r d . e d u /$ newsletters/harvard_mens_health_watch/ 2012/February
}

weight and BMI - that are derived from body weight and body height - which further has the advantage of being contactless, low-cost, unobtrusive, and unconstrained ${ }^{15}$.

Recently, BMI was estimated from face images by Wen and Guo [270]. In their work, the face was detected, normalized, and an active shape model was fitted, based on which, geometry and ratio features were extracted (cheekbone to jaw width, width to upper facial height ratio, perimeter to area ratio, eye size, lower face to face height ratio, face width to lower face height ratio and mean of eyebrow height), normalized and finally subjected to support vector regression. When training and testing using the MORPH-II database which contains 55,000 images, with variations in age and ethnicity - the method obtained MAEs ranging from $2 \mathrm{~kg} / \mathrm{m}^{2}$ ('normal weight') to $9.5 \mathrm{~kg} / \mathrm{m}^{2}$ ('obese'), where the higher errors generally related to medically atypical cases such as 'under weight' and 'obese' subjects. Prior to that, Velardo and Dugelay [260] had devised a method to estimate weight from manually measured body-anthropometric data, by constructing a weight prediction metric using multiple regression analysis. When training and testing using the NHANES dataset ${ }^{16}$ which contains data of 28,000 subjects - the results showed that weight could be estimated in $93 \%$ of the test data with an error of less than $10 \%$. Velardo et al. [261] presented a practical study with a weight estimation error of $4 \%$ on a self recorded Kinect RGBD dataset of 15 subjects, where body anthropometric measurements were extracted automatically. This work proceeded also to evaluate - based on the same measurements - the subjects' height and gender, using realtime reduced-complexity 3D model fitting. A related work for weight estimation by Labati et al. [134] studied frame sequences representing walking subjects, where different walking directions and lighting conditions were used to challenge the algorithms. Features such as body height, body volume (estimated by ellipses passing through the silhouettes), body shape and walking direction were extracted to train a neural network towards body weight estimation. The mean errors ranged from $-2.38 \mathrm{~kg}$ to $0.02 \mathrm{~kg}$ on a small proprietary dataset of 20 subjects.

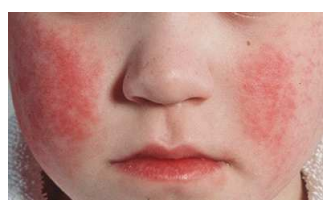

(a) Parvovirus

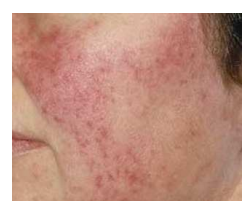

(b) Rosacea

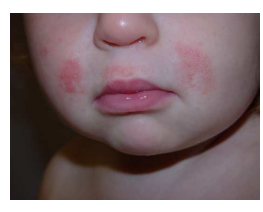

(c) Contact Dermatitis
Fig. 9. Examples of skin diseases. The human skin often exhibits symptoms of internal and external diseases.

\section{Health and skin lesions}

In addition to geometric body characteristics, another class of soft biometrics that relates to medical conditions is the

\footnotetext{
${ }^{15}$ Extracting BMI or body weight also offers other advantages, e.g., in forensics, as these measures can be sensed at a distance

${ }^{16} \mathrm{http}: / /$ www.cdc.gov/nchs/nhanes.htm
} 
To appear in IEEE Transactions on Information Forensics and Security (TIFS), 2015

human skin (see Figure 9). The main motivation here is clear; early detection of malignant skin lesions is very critical towards initiating early and effective cure methods. For cases like skin cancer - which is the most common cancer type, with 3.5 million cases of basal and squamous cell skin cancer diagnosed in the United States each year ${ }^{17}$ - early detection can allow for a suitable cure and even prevent death. While such automated systems cannot fully replace medical professionals, it is often noted that visual examination by an expert can be difficult (deeper subsurface inspection is required), while biopsies can be invasive and time consuming. As a result there have been substantial efforts to develop novel noninvasive optical imaging techniques as a way to detect and analyze skin lesions [222], [100], with recent advances involving smart hand-held devices [36], [263]. In addition to skin cancer, image-based methods can apply to other common skin diseases like chronic inflammatory psoriasis [149], [57], pressure ulcers [141] and hyper-pigmentation evolution [194].

b) Health and wrinkles: In addition to skin lesions, wrinkles can also reveal stress states that relate to the medical condition of an individual. Naturally wrinkles also carry discriminative clues, as was analyzed by Batool et al. [17], where a set of facial wrinkles extracted from images were treated as curve patterns towards finding similarity between subjects. In their work, different metrics based on Hausdorff distance and curve-to-curve correspondences were presented in an effort to quantify this similarity, and bipartite graph matching was employed for finding correspondences between curves from two patterns. The resulting recognition rates exceeded $65 \%$ at rank 1 and $90 \%$ at rank 4 , suggesting that a set of wrinkle patterns may be unique to an individual.

\section{Databases}

There is currently a limited number of publicly available biometric datasets with annotated health-information. This is partly due to the sensitive nature of medical data which raises several ethical and privacy concerns especially when the subject identity is divulged. Hence, studies on related topics often involve private medical datasets, accessible only by associates. The MORPH-II and the CAESAR datasets are exceptions, providing images which include weight information.

\section{MATERIAL, BEHAVIORAL, AND OTHER SOFT BIOMETRIC ATTRIBUTES}

We next consider objects that may be associated with a person (e.g. accessories such as hats and scarves), traits that have a strong behavioral utility, as well as other soft biometric traits that have received substantial attention lately. Some of these attributes - related to eye lenses, eye glasses, hats, scarfs, clothes, etc. - may not be immediately associated with biometrics in the classical sense; nonetheless they remain of high interest in identification and security applications, often due to their conspicuous nature. Some of these objects and attributes may naturally be associated with different utilities at once. Take for example the color of the clothes. This

${ }^{17}$ http://www.cancer.org/cancer/skincancer/index attribute, while heavily related to subject occlusion, can also assist in human recognition, as shown in the work by Denman et al. [46] who presented a video surveillance and tracking system that is based on the color of clothes, where specifically color histograms of the head, torso and legs were processed in order to re-identify individuals. The same attribute was used also by D'Angelo and Dugelay in [37] for analyzing the color of clothes in a sports-soccer scenario, with the aim of preventing fights between fans of opposing teams (shirt color can be a strong indicator of the team which a subject supports).

\section{A. Material characteristics}

Unlike most other attributes, this class consists of characteristics that can in fact hinder the recognition process. These include face occluding characteristics, such as scarfs, caps, hats and eye glasses. Such occluding accessories have received attention primarily for their ability to hinder person recognition or even human detection, from both machine and human perspectives. The consideration of such attributes in the context of face recognition, occlusion and occlusion detection - can be found in papers by Yoon and Kee [284] and Wen et al. [269]. In their work, occlusion detection is performed for specific facial features such as the mouth region, while some other works such as that by Lin and Liu [146], observe the face holistically, classifying it as occluded or not, by employing PCA and SVM. Below we review some of these material characteristics.

Scarfs are of particular importance because they are sometimes used by individuals in a crime spree. Min et al. [166] presented a scarf detection algorithm based on PCA and SVM, and reported a detection rate of about $99 \%$, on the ARFD database ([163]) which features 300 scarf-occluded and 300 not-occluded faces. This work was later used by Min et al. [168] towards face recognition, where Gabor wavelets, PCA and SVM were employed for occluded faces, while nonoccluded facial parts were computed by block-based LBP. This work used the AR face database ${ }^{18}$, and reported a recognition rate of $94.83 \%$ for non-occluded faces, and $92.08 \%$ for occluded faces.

In contrast to scarfs, which are mainly used to cover the lower part of the face, headgear can occlude the upper part of the face. Du et al. [53] studied the occlusion of hard hats in construction sites, and studied face detection using the Viola Jones algorithm, also analyzing the motion and color in the upper face region in a dataset of 5 video sequences. Similarly, cap detection was performed by Min and Dugelay [167] who utilized dynamic time warping (DTW) and agglomerative hierarchical clustering, to achieve an accuracy of $87.5 \%$ on a private dataset of 10 recorded subjects with and without caps in 40 videos.

Similarly, eye glasses and contact lenses can also have an occluding effect - albeit lesser than that of the previous two characteristics - and can interfere with the performance of iris and periocular-based recognition systems. In this context, the impact of contact lenses on iris recognition was examined by

\footnotetext{
${ }^{18}$ http://www-sipl.technion.ac.il/new/DataBases/Aleix $\backslash \% 20$ Face $\backslash$ $\% 20$ Database.htm
} 
Baker et al. [13]. Their experiments suggested that even noncosmetic prescription contact lenses can impact the matching accuracy of iris recognition. Subsequent research by Erdogan and Ross [56] established the possibility of automatically detecting such lenses in iris images. Glass detection in face images was studied by Jiang et al. in [114], [277], [272], with best results obtained using thermal images [101].

Makeup can also be viewed as an occluding characteristic, and has the potential to substantially alter the appearance of a face. Furthermore, makeup and cosmetics can be used to successfully alter or camouflage wrinkles, birth moles, scars and tattoos. The impact of makeup on face recognition has been studied by Dantcheva et al. [38], while Chen et al. [25] performed makeup detection, achieving a detection accuracy of approximately $91 \%$.

\section{B. Behavioral soft biometric traits}

Behavioral attributes are a relatively new class of soft biometric traits that can assist in detecting different human behaviors. For example, the accent of a person can be gleaned from human speech which can also convey ancillary information such as the gender of the speaker. The associated gender estimation and accent recognition can be fused with classical speech or speaker recognition methods in order to improve recognition performance. In this context, Deshpande et al. [48] showed promising results, where, focusing on American and Indian accents in the English language, they analyzed the second and third formant frequencies of specific accent markers and classified those based on Gaussian mixture models.

Some other behavioral traits that can be automatically gleaned from video and images include facial expressions which also carry supplementary biometric information. Interesting work can be found in related survey papers [288], [162], [224]. In the specific context of soft biometrics, Kashyap et al. [118] studied video segments of individuals encoded by a facial action coding system (FACS) for facial expression quantification. Related experiments studied the intensity of a number of facial units (each facial unit represents movement of a specific facial muscle), and confirmed the suitability of facial asymmetry, as well as of action unit combinations, towards person identification.

\section{Scars, marks, tattoos}

Scars, marks and tattoos are increasingly employed towards person recognition in forensics and law enforcement (cf. Spaun [240]). Such traits can be particularly useful in describing wanted or missing people, or even unidentified bodies, and the traits are often bundled together due to the high degree of distinctiveness they can jointly provide. Scars and marks are mainly defined by the location in which they occur, while tatoos carry information in their position, color, content (such as human forms, animals, flags, plants, words, and other objects), as well as the way in which they are 'imprinted' (chemical, branded, cut) into the skin (see ANSI/NIST standard report ${ }^{19}$ ). Despite the rich information

\footnotetext{
${ }^{19} \mathrm{http} / / /$ www.nist.gov/itl/ansi/upload/sp500-245-a16.pdf
}

that is embedded in tatoos and marks, there is substantial ambiguity associated with their classification. To overcome this ambiguity, Lee et al [142] proposed a content-based image retrieval system that uses SIFT features, reporting rank-20 retrieval accuracies of $98.6 \%$ on high resolution images (WebDB), and $77.2 \%$ accuracy on an operational database (MIDB). Furthermore, Jain and Park [112] [188] used AAM to locate and segment primary facial features (e.g., eyes, nose, and mouth) and employed Laplacian-of-Gaussian (LoG) and morphological operators in order to detect facial marks. Their algorithm was tested on a subset of the FERET database consisting of 426 images of 213 subjects, and showed a marginal improvement in rank-1 identification accuracy over the commercial FaceVACS face recognition system. A similar evaluation was conducted on the Mugshot-dataset having 1,225 images of 671 subjects, achieving an improvement in accuracy from $91.88 \%$ to $93.14 \%$. Along the same lines, Lin and Tang [145] used SIFT-based extraction and fusion of skin irregularities - such as facial marks - to improve face matching performance by about 5\% on the XM2VTS and HRDB database.

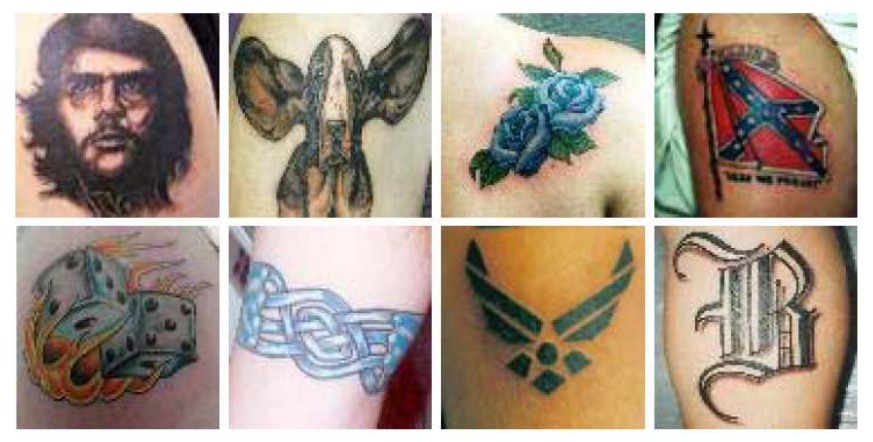

Fig. 10. Examples of different tattoos [142].

Additionally, Pierrard and Vetter [193], in the setting of face identification, proposed a method to extract moles using normalized cross correlation (NCC) matching and a morphable model. Experiments were conducted on the FERET database, and the authors claimed that their method was pose- and lighting- invariant (albeit computationally expensive) due to the underlying 3D morphable model. Srinivas et al. [241] used facial marks to differentiate between identical twins, while Nurhudatiana et al. [181] studied relatively permanent pigmented or vascular skin marks (RPPVSM) found on the backs of 269 male subjects, and employed this soft biometric trait in forensics.

1) Databases: XM2VTS database ${ }^{20}$. This is a multi-modal face database, corresponding to 295 subjects, where for each subject there are four recordings taken over a period of four months, and where each recording contains a speaking head shot and a rotating head shot. Sets of data taken from this database are available, including high quality color images, $32 \mathrm{KHz}$ 16-bit sound files, video sequences and a 3d Model. XM2VTS has been used for skin irregularity detection (e.g. facial marks). Mugshot [268] database. This database contains

\footnotetext{
${ }^{20} \mathrm{http} / / /$ www.ee.surrey.ac.uk/CVSSP/xm2vtsdb/
} 
To appear in IEEE Transactions on Information Forensics and Security (TIFS), 2015

face images of 1500 subjects. Each subject has two images: a frontal view and a profile view. The photographs are provided from archives of the FBI. The Mugshot dataset has been used in facial marks studies. Web-DB database ${ }^{21}$. The Webdownloaded Tattoo Image Database contains 4,323 tattoo images downloaded from the Web. Each image is of size $90 \times 90$. $M I-D B$ database. The Michigan State Police Tattoo Database contains tattoo images from suspects and convicts, recorded over a period of ten years, by the Michigan police department. Tattoos are photographed when a suspect / convict is booked, and assigned a keyword as defined in the ANSI/NIST standard and then stored in the database along with other demographic information. There are 69,507 operational tattoo images, each of size $640 \times 480$.

\section{OPEN RESEARCH PROBLEMS}

There are a number of open research problems in this expanding field.

a) Correlation between soft biometric traits: The issue of correlation is not well studied in the soft biometric literature. While Adjeroh et al. [2] investigated the correlation between geometric attributes of the human body, such a study has not been undertaken for other traits. Understanding the correlation structure between these traits will allow for the design of effective fusion schemes for combining them.

b) Defining the number of categories for a soft biometric trait: A main issue also relates to finding an efficient and robust way to create the corresponding categories for a particular trait. While for some traits, this categorization is straight forward, for other traits the division into categories can be complex and not immediately clear. For example gender recognition is easily viewed as a binary classification problem (male vs. female), while other traits such as age or race can be continuous and ambiguous in nature. The challenge is the fact that this classification must (at least partly) adhere to the semantic interpretation of traits (recall human compliance), as well as must account for the significant differences between actual traits and the human perceived version of these same traits.

In addition to the aforementioned challenges relating to the perceptual idiosyncracies of humans, there are also challenges relating to the limitations of algorithmic and hardware resources. In short, a soft biometric system and its classification must take into consideration the resources of the system. For instance, from an image processing point of view, trait categorization can be limited by the employed estimation algorithms as well as the quality of the input images. As a result, while a certain camera-and-algorithm system may be able to robustly differentiate between many categories, another may come short of that, hence introducing the need for careful consideration of resources on the degree of refinement of categories.

c) Automatic quality assessment of extracted soft biometric traits: On a similar note, another challenge is to reliably extract these traits. For instance, from an imageprocessing point of view, the challenge of reliable feature

\footnotetext{
${ }^{21}$ http://www.tattoodesign.com/gallery/
}

extraction is often related to ambient factors such as lighting. Illumination variation is a significant problem which, in many cases - especially in surveillance videos - can result in massive variations in color. At the same time, soft biometrics offer substantial flexibility as far as acquisition is concerned. For example, traits such as hair color, skin color, height, weight, gait and different facial measurements, can be sensed (and estimated) from both frontal as well as side views of a subject. So the challenge is to balance the flexibility in acquisition afforded by soft biometric traits (e.g., under different pose and expression changes) with variations due to sensing (e.g., illumination) by optimizing the number of categories that can be used for a particular soft biometric trait (e.g., number of discrete colors for clothing). Automatic assessment of data quality may be necessary for this reason.

d) Subjective perception by humans: One wide open challenge is to design soft biometric matching systems that account for human variability in describing different traits. The human compliance attributed to soft biometrics, while useful in many ways, also introduces problems relating to the broad variability in syntax and language when it comes to describing different soft biometric traits (e.g., hair can be described as "red", "auburn" to "brown" - see [30] for an interesting experiment on this). Furthermore, humans can be inaccurate when describing measurements [287], where descriptions can depend on the person's own attributes and their own perception of population averages and variation [209]. Early literature to overcome this include methods that apply reference examples, or that apply comparative / pairwise ranking (cf. [209]). For example, determining whether a person is taller than someone else is easier and more accurate than an absolute estimation of the person's height.

e) Fusion of soft biometrics with primary traits: Another topic is the incorporation of soft biometric traits into primary biometric systems. One could imagine a soft biometric system that quickly classifies and filters a database, and where this pruning is followed by a more robust but slower classical biometric search. In this setting, the challenge is to design and fuse the component systems in a way that satisfies the specific speed and reliability requirements of the overall system.

f) Statistical modeling and mathematical analysis of soft biometric systems: It is necessary to provide a mathematical analysis of the limits and capabilities of soft biometric systems. This need is accentuated by the fact that such systems are bound to get bigger and more complex (by considering numerous biometric and soft biometric traits), as well as more demanding. Systems may be required to process an abundance of information pertaining to large populations (e.g., over 1.2 Billion subjects in the UIDAI biometrics project ${ }^{22}$ ). Analysis of complex soft biometric systems is necessary; while simulations and empirical studies can often provide some insight into the limits of such systems, empirical approaches may not be able to accurately characterize the performance of large-scale systems with increased complexity.

One related problem that has remained largely unexplored is that of estimating the reliability of person recognition using

${ }^{22}$ http://uidai.gov.in/ 
soft biometrics. Such analysis would have to consider the effect of the population statistics, as well as have access to mathematical models for the sensing and algorithmic capabilities of the system. This can help in automatically establishing the number of categories that are necessary for a soft biometric trait in order to guarantee a certain degree of performance. Further, it may be instructive to derive theoretical bounds on the discriminative and classification capabilities of a soft biometric system. Very preliminary work on this can be found in [40].

Along the same lines, it would be worthwhile to predict the expected improvement in matching accuracy and reduction in computational complexity when utilizing soft biometric traits as filters on large databases. Such analysis would be beneficial in applications such as time-constrained human identification in video surveillance systems. The analysis should take into account the implicit errors in soft biometric classification, since these errors would affect the overall identification performance. A very early analysis can be found in the work by Dantcheva et al. [41].

g) Spoofing of soft biometrics: The potential of using soft biometrics in several sensitive commercial and security applications, has to be balanced with understanding and minimizing the vulnerability of such traits to obfuscation (where an individual camouflages their soft biometric traits) and spoofing (where an individual attempts to look like another person in terms of their soft biometric traits). This is particularly important because the very nature of soft biometrics allows for easier spoofing than classical biometric traits; indeed one can easily imagine how gender, age and ethnicity can be disguised simply by styling and cosmetics. Similarly, eye color can be spoofed with color lenses, hair can be dyed, and skin color - and the overall facial appearance - can be modified by makeup. In this regard, the use of cosmetic products towards soft biometric spoofing would be a realistic threat. Early work on this can be found in [26], where the impact of makeup on gender- and age- estimation algorithms has been studied. Specifically the authors in this work discuss gender spoofing (see Figure 11) where male subjects attempt to look like females and vice versa, as well as discuss age alteration where female subjects attempt to look younger or older than they actually are. Experimental results with several genderand age-estimation algorithms, suggested that gender and age estimation systems can be impacted by the application of facial makeup. Additional proof of the impact of makeup on age has been reported in [61], thus further accentuating the open challenge of designing algorithms that are less susceptible to spoofing and obfuscation techniques.

h) Ethical issues: In addition to the mainly algorithmic challenges mentioned above, there exist other challenges in the area of soft biometrics, including ethical challenges related to the use of skin color, ethnicity and gender in recognition systems (see [170]). Ethical issues might also rise as a result of the fact that soft biometrics can be covertly extracted from data collected for other purposes. At the same time, soft biometrics do a better job at preserving privacy, given that the stored signatures are often less descriptive than, say, a high resolution image of an iris. The challenge is to properly traverse this thin

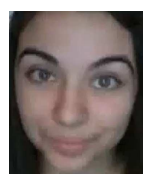

(a)

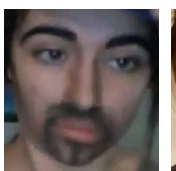

(e)

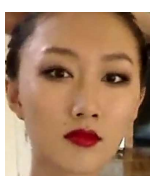

(b)

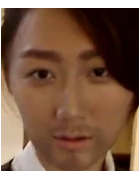

(f)

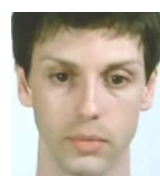

(c)

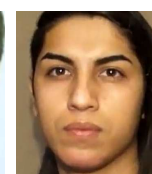

(d)

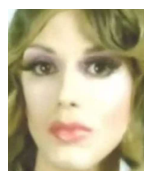

(g)

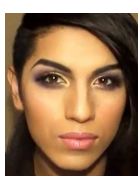

(h)
Fig. 11. Example images for gender spoofing [26]: female-to-male subset: female subjects apply makeup to look like males (a) $\rightarrow$ (e), (b) $\rightarrow$ (f); maleto-female subset: male subjects apply makeup to look like females $(\mathrm{c}) \rightarrow(\mathrm{g})$, $(d) \rightarrow(h)$. The images in the upper row are before the application of makeup and the ones below are the corresponding images after makeup.

line between privacy enhancement and privacy infringement.

i) Privacy implications: In the context of biometrics, privacy refers to the assurance that the biometric data collected from an individual is not used to deduce any type of information about the individual [123], i.e., it should be used only for matching purposes. However, as discussed in this article, biometric data offer additional information about an individual which can be automatically deduced. This can be viewed as privacy leakage since an entity can learn additional information about a person (or population) from the stored data, without receiving authorization from the person for such a disclosure. Therefore, it is necessary to ensure that biometric data stored in a system are used only for the intended purpose and not for purposes that may result in a 'function creep'. This has heightened the need to develop differential privacy constructs, where the biometric data can only reveal certain attributes (e.g., gender) while other attributes (e.g., ethnicity) are suppressed. The term "de-identification" has often been used in this context.

De-identification has become an essential keyword, since a large amount of video surveillance systems have been employed in public spaces. Such surveillance unintentionally invades the privacy of individuals captured in videos. To circumvent such a privacy invasion, subjects in the video can be de-identified. De-identification refers to the obfuscation of the identity of an individual without obscuring the performed action, see [3]. For maintaining the purpose of video surveillance, limited information related to subject and action has to be kept. Gender, ethnicity, body height, color of clothes and color of hair may adequately serve as remaining evidence. In [259] the authors present a privacy preserving video surveillance system, where subjects are de-identified and the remaining information includes the subject height, weight and color of clothes. Specifically, this approach uses body height, weight and clothes color to track a person inside a building under surveillance, and to display the position of this person on a monitor, in the form of a square placed within the map of the building. The square displayed in the monitor is properly colored; the upper part of the square has the color of the clothing of the upper body of the subject, while the lower 
part of the square is colored to match that of the clothes on the lower part of the body.

On the other hand, de-identification of soft biometrics, while retaining the facial identity [183] can be useful for extending different levels of privacy to a face image in a central database.

\section{Input image Transformed images}
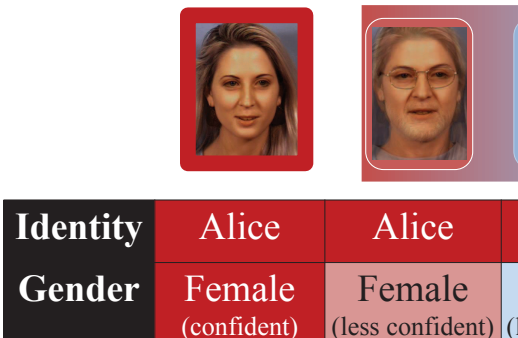

\begin{tabular}{c|c|c|c} 
Alice & Alice & Alice & Alice \\
$\begin{array}{c}\text { Female } \\
\text { (confident) }\end{array}$ & $\begin{array}{c}\text { Female } \\
\text { (less confident) }\end{array}$ & $\begin{array}{c}\text { Male } \\
\text { (less confident) }\end{array}$ & $\begin{array}{c}\text { Male } \\
\text { (confident }\end{array}$ \\
\hline
\end{tabular}

Fig. 12. Perturbing gender information in face images by Othman and Ross [183]. The soft biometric attribute of a face (e.g., gender) is suppressed, while preserving the ability of the face matcher to recognize the individual. This ensures that the stored biometric data is not used for purposes beyond what was expressed during the time of data collection.

j) Standardization: ANSI/NIST-ITL 1-2011 (Data Format for the Interchange of Fingerprint Facial, \& Other Biometric Information - Part 1) suggests "additional descriptive information" such as eye patch, clear glasses, dark glasses, head covering, scarf, moustache, beard, eye color, hair color. Further, the standard "American National Standard for Information Systems- Data Format for the Interchange of Fingerprint, Facial, \& Scar Mark \& Tattoo (SMT) Information" widely covers the use and classification of scars, marks and tattoos. Further standardization will be essential to balance privacy and utility.

\section{CONCLUSIONS}

A review of the biometric literature suggests that research in soft biometrics is on the rise. This expansion is due to the large number of applications that can benefit from the extraction of soft biometric traits. Examples of such applications include security, surveillance, retrieval, and health care. In this article, we reviewed some of the methods that have been developed for extracting soft biometric attributes from image, video and audio data. We also introduced a taxonomy to organize the various soft biometric traits that have been presented in the biometric literature. Further, we discussed the benefits and limitations of different soft biometric approaches. Finally, we discussed some of the open research problems in soft biometrics research.

We believe that the main advantage of soft biometric traits lies in their ability to describe people from a human perspective. Therefore, it bridges the gap between machine and human descriptions of a person. We also believe that the performance of soft biometric systems needs to be considered carefully, as it can be affected significantly by several factors such as sensing and feature extraction. By carefully investigating the accuracy, reliability and distribution of different soft biometric traits, it is possible to judiciously use them in large-scale biometric systems. However, it is necessary to be cognizant of the privacy implications of using soft biometric traits. By balancing privacy with performance, it is likely that soft biometric traits will have a critical role to play in next generation identification systems.

\section{REFERENCES}

[1] M. C. Da Costa Abreu and M. Fairhurst. Enhancing identity prediction using a novel approach to combining hard-and soft-biometric information. IEEE Trans. on System, Man, and Cybernetics Part C, 41(5):599-607, 2011.

[2] D. Adjeroh, D. Cao, M. Piccirilli, and A. Ross. Predictability and correlation in human metrology. In Proc. of the IEEE International Workshop on Information Forensics and Security, 2010.

[3] P. Agrawal and P. J. Narayanan. Person de-identification in videos. IEEE Trans. on Circuits and Systems for Video Technology, 21/3, 2011.

[4] H. Ailisto, M. Lindholm, S.-M. Mkel, and E. Vildjiounaite. Unobtrusive user identification with light biometrics. In Proc. of NordiCHI, 2004.

[5] A. M. Alberta, K. Ricanek, and E. Patterson. A review of the literature on the aging adult skull and face: implications for forensic science research and applications. Forensic Science International, 172(1):1-9, 2007.

[6] B. Allen, B. Curless, and Z. Popovic. The space of human body shape: reconstruction and parameterization. ACM Trans. on Graphics, 22(3):587-594, 2003.

[7] T. R. Alley. Social and applied aspects of perceiving faces. Lawrence Erlbaum Association, 1988.

[8] G. Amayeh, G. Bebis, and M. Nicolescu. Gender classification from hand shape. In Proc. of IEEE Conference on Computer Vision and Pattern Recognition Workshops, 2008.

[9] N. Angier. Do races differ? not really, genes show. The New York Times, Aug. 22, 2000.

[10] B. M. Auerbach. Methods for estimating missing human skeletal element osteometric dimensions employed in the revised fully technique for estimating stature. American Journal of Physical Anthropology, 145(1):67-80, 2011.

[11] J. A. Bachorowski and M. J. Owren. Acoustic correlates of talker sex and individual talker identity are present in a short vowel segment produced in running speech. Journal of Acoustic Society Americas, 106(2):1054-1063, 1999.

[12] A. Badawi, M. Mahfouz, R. Tadross, and R. Jantz. Fingerprint-based gender classification. In Proc. of International Conference on Image Processing, Computer Vision and Pattern Recognition, pages 41-46, 2006.

[13] Sarah E. Baker, Amanda Hentz, Kevin W. Bowyer, and Patrick J. Flynn. Degradation of iris recognition performance due to noncosmetic prescription contact lenses. Computer Vision and Image Understanding, 114(9):1030 - 1044, 2010.

[14] Alexandru O. Balan and Michal J. Black. The naked truth: estimating body shape under clothing. In Proc. of European Conference on Computer Vision, 2008.

[15] S. Baluja and H. A. Rowley. Boosting sex identification performance. International Journal of Computer Vision, 71:111-119, 2006.

[16] A. Bansal, R. Agarwal, and R. K. Sharma. Predicting gender using iris images. Research Journal of Recent Sciences, 3(4):20-26, April 2014.

[17] N. Batool, S. Taheri, and R. Chellappa. Assessment of facial wrinkles as a soft biometrics. In Proc. of IEEE International Conference on Automatic Face and Gesture Recognition, 2013.

[18] J. Bekios-Calfa, J. M. Buenaposada, and L. Baumela. Revisiting linear discriminant techniques in gender recognition. IEEE Trans. on Pattern Analysis and Machine Intelligence, 33(4):858-864, 2011.

[19] J. Bekios-Calfa, J. M. Buenaposada, and L. Baumela. Robust gender recognition by exploiting facial attributes dependencies. Pattern Recognition Letters, 2013.

[20] C. BenAbdelkader and L. Davis. Estimation of anthropomeasures from a single calibrated camera. In Proc. of IEEE International Conference on Automatic Face and Gesture Recognition, pages 499-504, 2006.

[21] L. Bourdev, S. Maji, and J. Malik. Describing people: A poseletbased approach to attribute classification. In Proc. of International Conference on Computer Vision , 2011.

[22] D. Cao, C. Chen, M. Piccirilli, D. Adjeroh, T. Bourlai, and A. Ross. Can facial metrology predict gender? In Proc. of International Joint Conference on Biometrics, 2011.

[23] L. Cao, M. Dikmen, Y. Fu, and T. S. Huang. Gender recognition from body. In Proc. of ACM Multimedia, 2008. 
[24] J. Carnicky and J. D. Chorvat. Three-dimensional measurement of human face with structured-light illumination. Measurement science review, 6:1-4, 2006.

[25] C. Chen, A. Dantcheva, and A. Ross. Automatic facial makeup detection with application in face recognition. In Proc. of IAPR International Conference on Biometrics, 2013.

[26] C. Chen, A. Dantcheva, and A. Ross. Impact of facial cosmetics on automatic gender and age estimation algorithms. In Proc. of International Conference on Computer Vision Theory and Applications, 2014

[27] C. Chen and A. Ross. Evaluation of gender classification methods on thermal and near-infrared face images. In Proc. of International Joint Conference on Biometrics, 2011.

[28] K. Chen, S. Gong, T. Xiang, and C. C. Loy. Cumulative attribute space for age and crowd density estimation. In Computer Vision and Pattern Recognition (CVPR), 2013 IEEE Conference on, pages 2467-2474. IEEE, 2013.

[29] L. Chen, Y. Wang, and Y. Wang. Gender classification based on fusion of weighted multi-view gait component distance. In IEEE Chinese Conference on Pattern Recognition, 2009.

[30] N. Chhaya and T. Oates. Joint inference of soft biometric features. In Proc. of IAPR International Conference on Biometrics, 2012.

[31] D. G. Childers and K. Wu. Gender recognition from speech. part II: Fine analysis. Journal of the Acoustical Society of America, 90:1841$1856,1991$.

[32] B. A. Chiraz, R. Cutler, and L. Davis. View-invariant estimation of height and stride for gait recognition. In Proc. of International Conference on Biometric Authentication, volume 2359/2006, pages 155-167, 2002.

[33] T. Cootes, G. Edwards, and C. Taylor. Active appearance models. IEEE Trans. on Pattern Analysis and Machine Intelligence, 23(6):681-685, 2001

[34] S. Crihalmeanu, A. Ross, S. Schuckers, and L. Hornak. A protocol for multibiometric data acquisition, storage and dissemination. Technical report, WVU, 2007.

[35] A. Criminisi, I. Reid, and A. Zisserman. Single view metrology. International Journal of Computer Vision, 40(2):123-148, 2000.

[36] B. D'Alessandro and A. P. Dhawan. 3-d volume reconstruction of skin lesions for melanin and blood volume estimation and lesion severity analysis. IEEE Trans. on Medical Imaging, 31(11):2083-2092, 2012.

[37] A. D'Angelo and J.-L. Dugelay. People re-identification in camera networks based on probabilistic color histograms. In Proc. of IS \&amp; T/SPIE Electronic Imaging: Science and Technology, pages 78820K78820K. International Society for Optics and Photonics, 2011.

[38] A. Dantcheva, C. Chen, and A. Ross. Can facial cosmetics affect the matching accuracy of face recognition systems? In Proc. of BTAS, 2012.

[39] A. Dantcheva, J.-L. Dugelay, and P. Elia. Person recognition using a bag of facial soft biometrics (BoFSB). In Proc. of IEEE International Workshop on Multimedia Signal Processing, 2010.

[40] A. Dantcheva, J.-L. Dugelay, and P. Elia. Soft biometric systems: reliability and asymptotic bounds. In Proc. of IEEE International Conference on Biometrics: Theory, Applications and Systems, 2010.

[41] A. Dantcheva, A. Singh, P. Elia, and J.-L. Dugelay. Search pruning in video surveillance systems: Efficiency-reliability tradeoff. In Proc. of International Conference on Computer Vision Workshops, 2011.

[42] A. Dantcheva, C. Velardo, A. D’Angelo, and J.-L. Dugelay. Bag of soft biometrics for person identification. New trends and challenges. Multimedia Tools and Applications, 51:739-777, 2011.

[43] D. De Angelis and R. Sala. New method for height estimation of subjects represented in photograms taken from video surveillance systems. Journal of Forensic and Legal Medicine, 121:489-492, 2007.

[44] B. DeCann and A. Ross. Gait curves for human recognition, backpack detection, and silhouette correction in a nighttime environment. In Proc. of SPIE Conference on Biometric Technology for Human Identification VII, 2010.

[45] M. Demirkus, K. Garg, and S. Guler. Automated person categorization for video surveillance using soft biometrics. In SPIE Defense, Security, and Sensing, pages 76670P-76670P, 2010.

[46] S. Denman, A. Bialkowski, C. Fookes, and S. Sridharan. Determining operational measures from multi-camera surveillance systems using soft biometrics. In Proc. of IEEE International Conference on Advanced Video and Signal based Surveillance, 2011.

[47] S. Denman, C. Fookes, A. Bialkowski, and S. Sridharan. Softbiometrics: Unconstrained authentication in a surveillance environment. In Proc. of International Conference on Digital Image Computing: Techniques and Applications, pages 196-203, 2009.
[48] S. Deshpande, S. Chikkerur, and V. Govindaraju. Accent classification in speech. In Proc. of IEEE AutoID, pages 139-143, 2005.

[49] P. Dias, D. Santos, D. Souza, H. Santos, C. Coelho, M. T. Ferreira, E. Cunha, and B. Sousa S. A new approach for 3d craniometric measurements using $3 \mathrm{~d}$ skull models. In Proc. of Information Visualisation (IV), pages 462-467. IEEE, 2013.

[50] H. Ding, D. Huang, Y. Wang, and L. Chen. Facial ethnicity classification based on boosted local texture and shape descriptions. In Proc. of International Conference on Automatic Face and Gesture Recognition, pages 1-6, April 2013.

[51] L. Ding and A. M. Martinez. Precise detailed detection of faces and facial features. In Proc. of IEEE Conference on Computer Vision and Pattern Recognition, 2008.

[52] M. Douze, A. Ramisa, and C. Schmid. Combining attributes and fisher vectors for efficient image retrieval. In Proc. of IEEE Conference on Computer Vision and Pattern Recognition, pages 745-752, 2011.

[53] S. Du, M. Shehata, and W. Badawy. Hard hat detection in video sequences based on face features, motion and color information. In Proc. of International Conference on Computer Research and Development, 2011.

[54] B. Edelman, D. Valentin, and H. Abdi. Sex classification of face areas: how well can a linear neural network predict human performance? Biological systems, 4, 1996.

[55] L. El Shafey, E. Khoury, and S. Marcel. Audio-visual gender recognition in uncontrolled environment using variability modeling techniques. In Proc. of International Joint Conference on Biometrics, pages 1-8, 2014.

[56] G. Erdogan and A. Ross. Automatic detection of non-cosmetic soft contact lenses in ocular images. In Proc. of SPIE 8712, 2013.

[57] M. H. Ahmad Fadzil, D. Ihtatho, A. Mohd Affandi, and S. H. Hussein. Objective assessment of psoriasis erythema for pasi scoring. Journal of medical engineering \& technology, 33(7):516-524, 2009.

[58] A. B. Falsetti. Sex assessment from metacarpals of the human hand. Journal of Forensic Sciences, 40:774776, 1995.

[59] G. Farinella and J.-L. Dugelay. Demographic classification: Do gender and ethnicity affect each other? In Proc. of International Conference on Informatics, Electronics \& Vision, 2012.

[60] L. Farkas. Anthropometry of the head and face. Raven Press, 1994.

[61] R. Feng and B. Prabhakaran. Quantifying the makeup effect in female faces and its applications for age estimation. In Proc. of IEEE International Symposium on Multimedia, pages 108-115, 2012.

[62] V. Ferrari and A. Zisserman. Learning visual attributes. In Proc. of Conference on Neural Information Processing Systems, 2007.

[63] JM Ferryman. Pets 2007 video database. In Proc. of Privacy Enhancing Technologies Symposium, pages 1-103, 2007.

[64] D. Freire-Obregon, M. Castrillon-Santana, E. Ramon-Balmaseda, and J. Lorenzo-Navarro. Automatic clothes segmentation for soft biometrics. In Proc. of International Conference on Image Processing, 2014.

[65] S. Fu, H. He, and Z. Hou. Learning race from face: A survey. IEEE Trans. on Pattern Analysis and Machine Intelligence, 36(12):24832509, 2014.

[66] S.-Y. Fu, G.-S. Yang, and X.-K. Kuai. A spiking neural network based cortex-like mechanism and application to facial expression recognition. Computational Intelligence and Neuroscience, 2012(946589), 2012.

[67] Y. Fu, G. Guo, and T. S. Huang. Soft biometrics for video surveillance. Intelligent Video Surveillance: Systems and Technology, pages 407432, 2009.

[68] Y. Fu, G. Guo, and T. S. Huang. Age synthesis and estimation via faces: a survey. IEEE Trans. Pattern Analysis and Machine Intelligence, 32(11):1955-1976, 2010.

[69] Y. Fu and T. S. Huang. Human age estimation with regression on discriminative aging manifold. IEEE Trans. Multimedia, 10(4):578584, 2008.

[70] Y. Fu and T. S. Huang. Human age estimation with regression on discriminative aging manifold. IEEE Trans. on Multimedia, 10(4):578584, 2008

[71] Y. Fu, Y. Xu, and T.S. Huang. Estimating human ages by manifold analysis of face pictures and regression on aging features. In Proc. of IEEE International Conference on Multimedia \& Expo, pages 13831386, 2007.

[72] W. Gao and H. Ai. Face gender classification on consumer images in a multiethnic environment. In Proc. IEEE International Conference on Biometrics, pages 169-178, 2009.

[73] N. S. Gavrilova and L. A. Gavrilov. Genetics: Ethnicity. Encyclopedia of Aging, 2:538-539, 2002. 
[74] X. Geng, Z.-H. Zhou, and K. Smith-Miles. Automatic age estimation based on facial aging patterns. IEEE Trans. on Pattern Analysis and Machine Intelligence, 29(12):2234-2240, 2007.

[75] X. Geng, Z.-H. Zhou, Y. Zhang, G. Li, and H. Dai. Learning from facial aging patterns for automatic age estimation. In Proc. of $A C M$ Multimedia, pages 307-316, 2006.

[76] A. El Kissi Ghalleb, S. Sghaier, and N. E. Ben Amara. Face recognition improvement using soft biometrics. In Proc. of SSD, pages 1-6, 2013.

[77] P. Gnanasivam and S. Muttan. Fingerprint gender classification using wavelet transform and singular value decomposition. arXiv preprint arXiv:1205.6745, 2012.

[78] A. Godil and S. Ressler. Retrieval and clustering from a 3D human database based on body and head shape. In arXiv preprint, volume $1105.2800,2006$

[79] B. A. Golomb, D. T. Lawrence, and T. J. Sejnowski. Sexnet: a neural networkidentifies sex fromhuman faces. Advances in Neural Information Processing Systems, pages 572-577, 1990.

[80] E. Gonzalez-Sosa, R. Vera-Rodriguez, J. Fierrez, and J. Ortega-Garcia. Comparison of Body Shape Descriptors for Biometric Recognition Using MMW Images. In Proc. of International Conference on Pattern Recognition, pages 124-129, 2014.

[81] G. Guerra-Filho and A. Biswas. Image and Vision Computing, 30(3):251-261, 2012.

[82] G. Guo. Soft biometrics from face images using support vector machines. In Y. Ma and G. Guo, editors, Support Vector Machines Applications, pages 269-302. Springer International Publishing, 2014.

[83] G. Guo, C. Dyer, Y. Fu, and T. Huang. Is gender recognition affected by age? In Proc. of International Conference on Computer Vision Workshops, pages 2032-2039, 2009.

[84] G. Guo, Y. Fu, C. Dyer, and T.S. Huang. Image-based human age estimation by manifold learning and locally adjusted robust regression. IEEE Trans. on Image Processing, 17(7):1178-1188, 2008.

[85] G. Guo, Y. Fu, T. S. Huang, and C. Dyer. Locally adjusted robust regression for human age estimation. In Proc. of IEEE Workshop on Applications of Computer Vision, 2008.

[86] G. Guo, Y. Fu, T. S. Huang, and C. Dyer. A probabilistic fusion approach to human age prediction. In Proc. of IEEE Conference on Computer Vision and Pattern Recognition Workshops, 2008.

[87] G. Guo and G. Mu. A study of large-scale ethnicity estimation with gender and age variations. In Proc. of IEEE Conference on Computer Vision and Pattern Recognition Workshops, pages 79-86, june 2010.

[88] G. Guo, G. Mu, Y. Fu, C. Dyer, and T. S. Huang. A study on automatic age estimation using a large database. In Proc. of International Conference on Computer Vision, 2009.

[89] G. Guo, G. Mu, Y. Fu, and T. S. Huang. Human age estimation using bio-inspired features. In Proc. of IEEE Conference on Computer Vision and Pattern Recognition, pages 112-119, 2009.

[90] G. D. Guo. Age estimation and sex classification. Video Analytics for Business Intelligence, pages 101-131, 2012.

[91] S. Gupta and A. P. Rao. Fingerprint based gender classification using discrete wavelet transform \& artificial neural network. International Journal of Computer Science and Mobile Computing, 3(4):1289-1296, 2014.

[92] S. Gutta, J. Phillips, and H. Wechsler. Gender and ethnic classification of face images. In Proc. of IEEE International Conference on Automatic Face and Gesture Recognition, pages 194-199, 1998.

[93] M. Halstead, S. Denman, S. Sridharan, and C. Fookes. Locating people in video from semantic descriptions: A new database and approach. In Proc. of International Conference on Pattern Recognition, pages 45014506, Aug 2014

[94] H. Han and A. K. Jain. Age, gender and race estimation from unconstrained face images. MSU Technical Report, MSU-CSE-14-5, 2014.

[95] H. Han, C. Otto, and A. K. Jain. Age estimation from face images: Human vs. machine performance. In Proc. of International Conference on Biometrics, 2013.

[96] H. Han, C. Otto, X. Liu, and A.K. Jain. Demographic estimation from face images: Human vs. machine performance. IEEE Trans. on Pattern Analysis and Machine Intelligence, 37(6):1148-1161, June 2015.

[97] X. Han, H. Ugail, and I. Palmer. Gender classification based on 3D face geometry features using SVM. In Proc. of International Conference on CyberWorlds, 2009.

[98] N. Hasler, C. Stoll, B. Rosenhahn, T. Thormahlen, and H.-P. Seidel Estimating body shape of dressed humans. Computers \& Graphics, 33(3):211-216, 2009.
[99] R. Hauser, J. Smoliński, and T. Gos. The estimation of stature on the basis of measurements of the femur. Forensic science international, 147(2):185-190, 2005

[100] R. Hennessy, S. Bish, J.W. Tunnell, and M. K. Markey. Segmentation of diffuse reflectance hyperspectral datasets with noise for detection of melanoma. In Proc. of International Conference of the IEEE in Medicine and Biology Society, pages 1482-1485, 2012.

[101] J. Heo, S. G. Kong, B. R. Abidi, and M. A. Abidi. Fusion of visual and thermal signatures with eyeglass removal for robust face recognition. In Proc. of IEEE Conference on Computer Vision and Pattern Recognition Workshops, page 122, 2004

[102] J. Heo and M. Savvides. Gender and ethnicity specific generic elastic models from a single $2 \mathrm{~d}$ image for novel $2 \mathrm{~d}$ pose face synthesis and recognition. IEEE Trans. on Pattern Analysis and Machine Intelligence, 34(12):2341-2350, 2012.

[103] L. Hong, A. K. Jain, and S. Pankanti. Can multibiometrics improve performance? In Proc. of IEEE AutoID, volume 99, pages 59-64, 1999.

[104] S. Hosoi, E. Takikawa, and M. Kawade. Ethnicity estimations with facial images. In Proc. of IEEE International Conference on Automatic Face and Gesture Recognition, pages 195-200, 2004.

[105] S. D. Hu, B. Jou, A. Jaech, and M. Savvides. Fusion of region-based representations for gender identification. In Proc. of International Joint Conference on Biometrics, 2011.

[106] M. Ichino and Y. Yamazaki. Soft biometrics and its application to security and business. In Proc. of Workshop on Biometrics and Kansei Engineering, pages 314-319. IEEE, 2013.

[107] F. Introna Jr, G. Di Vella, and S. Petrachi. Determination of height in life using multiple regression of skull parameters. Bollettino della Societa italiana di biologia sperimentale, 69(3):153-160, 1993.

[108] A. Jain and J. Huang. Integrating independent components and linear discriminant analysis for gender classification. In Proc. IEEE International Conference on Automatic Face and Gesture Recognition, pages $159-163,2004$.

[109] A. K. Jain, S. C. Dass, and K. Nandakumar. Can soft biometric traits assist user recognition? In Proc. of SPIE, volume 5404, pages 561-572, 2004.

[110] A. K. Jain, S. C. Dass, and K. Nandakumar. Soft biometric traits for personal recognition systems. In Proc. of International Conference on Biometric Authentication, 2004

[111] A. K. Jain, P. J. Flynn, and A. Ross. Handbook of Biometrics. Springer, 2007

[112] A. K. Jain and U. Park. Facial marks: Soft biometric for face recognition. In Proc. of IEEE International Conference on Image Processing, volume 1, pages 37-40, 2009.

[113] S. Jia and N. Cristianini. Learning to classify gender from four million images. Pattern Recognition Letters, 58:35-41, 2015.

[114] X. Jiang, M. Binkert, B. Achermann, and H. Bunke. Towards detection of glasses in facial images. PAA, 3:9-18, 2000.

[115] A. Johnson and A. Bobick. Gait recognition using static activityspecific parameters. In Proc. of IEEE Conference on Computer Vision and Pattern Recognition, 2001.

[116] T. Kanchan and K. Krishan. Anthropometry of hand in sex determination of dismembered remains - a review of literature. Journal of Forensic and Legal Medicine, 18:14-17, 2011.

[117] T. Kanno, M. Akiba, Y. Teramachi, H. Nagahashi, and T. Agui. Classification of age group based on facial images of young males by using neural networks. Trans. on Information and Systems, E84D(8):1094-1101, 2001.

[118] A. L. Kashyap, S. Tulyakov, and V. Govindaraju. Facial behavior as a soft biometric. In Proc. of IAPR International Conference on Biometrics, 2012.

[119] A. Khan, A. Majid, and A. M. Mirza. Combination and optimization of classifiers in gender classification using genetic programming. Int J. Knowl.-Based Intell. Eng. Syst., 9:1-11, 2005.

[120] S. A. Khan, M. Nazir, S. Akram, and N. Riaz. Gender classification using image processing techniques: a survey. In Proc. of INMIC, 2011.

[121] A. Khotanzad and Y. Hong. Invariant image recognition by zernike moments. IEEE Trans. on Pattern Analysis and Machine Intelligence, 12:489-498, 1990.

[122] H.-C. Kim, D. Kim, Z. Ghahramani, and S. Y. Bang. Appearance-based gender classification with gaussian processes. Pattern Recognition Letters, 27:618-626, 2006.

[123] E. Kindt. Privacy and data protection issues of biometric applications. Law, Governance and Technology Series, 12, 2013.

[124] B. Klare and A. K. Jain. On a taxonomy of facial features. In Proc. of IEEE International Conference on Biometrics: Theory, Applications and Systems, pages 1-8, 2010. 
[125] Joshua C. Klontz and Anil K. Jain. A case study on unconstrained facial recognition using the boston marathon bombings suspects. Technical Report, (MSU-CSE-13-4), 2013.

[126] K. Krishan. Anthropometry in forensic medicine and forensic science'forensic anthropometry'. The Internet Journal of Forensic Science, 2(1):95-97, 2007.

[127] K. Krishan, T. Kanchan, and A. Sharma. Sex determination from hand and foot dimensions in a North Indian population. Journal of Forensic Sciences, 56(2):453-459, 2011.

[128] W. M. Krogman and M. Y. Iscan. The human skeleton in forensic medicine. Charles C. Thomas Springfield, 1986.

[129] N. Kumar, P. N. Belhumeur, and S. K. Nayar. Facetracer: a search engine for large collections of images with faces. In Proc. of European Conference on Computer Vision, 2008.

[130] N. Kumar, A. C. Berg, P. N. Belhumeur, and S. K. Nayar. Attribute and simile classifiers for face verification. In Proc. of International Conference on Computer Vision, 2009.

[131] N. Kumar, A. C. Berg, P. N. Belhumeur, and S. K. Nayar. Describable visual attributes for face verification and image search. IEEE Trans. on Pattern Analysis and Machine Intelligence, 33(10):1962-1977, 2011.

[132] Y. Kwon and N. Lobo. Age classification from facial images. In Proc. of IEEE Conference on Computer Vision and Pattern Recognition, pages 762-767, 1994.

[133] Y. Kwon and N. Lobo. Age classification from facial images. Computer Vision and Image Understanding, 74(1):1-21, 1999.

[134] R. D. Labati, A. Genovese, V. Piuri, and F. Scotti. Weight estimation from frame sequences using computational intelligence techniques. In Proc. of International Conference on Computational Intelligence for Measurement Systems and Applications, 2012.

[135] S. Lagree and K. W. Bowyer. Predicting ethnicity and gender from iris texture. In Proc. of IEEE Symposium on Technologies for Homeland Security, 2011.

[136] N. Lakshmiprabha, J. Bhattacharya, and S. Majumder. Age estimation using gender information. Computer Networks and Intelligent Computing, pages 211-216, 2011.

[137] C. H. Lampert, H. Nickisch, and S. Harmeling. Learning to detect unseen object classes by between-class attribute transfer. In Proc. of IEEE Conference on Computer Vision and Pattern Recognition, pages 951-958, 2009.

[138] A. Lanitis, C. Draganova, and C. Christodoulou. Comparing different classifiers for automatic age estimation. IEEE Trans. on System, Man, and Cybernetics Part C, 34(1):621-628, 2004.

[139] A. Lanitis, C. Taylor, and T. Cootes. Toward automatic simulation of aging effects on face images. IEEE Trans. on Pattern Analysis and Machine Intelligence, 24(4):442-455, 2002.

[140] R. A. Lazenby. Identification of sex from metacarpals: effect of side asymmetry. Journal of Forensic Sciences, 39:11881194, 1994.

[141] J. Leachtenauer, S. Kell, B. Turner, C. Newcomer, C. Lyder, and M. Alwan. A non-contact imaging-based approach to detecting stage i pressure ulcers. In Proc. of International Conference of the IEEE in Medicine and Biology Society, pages 6380-6383. IEEE, 2006.

[142] J.-E. Lee, A. K. Jain, and R. Jin. Scars, marks and tattoos (smt): Soft biometric for suspect and victim identification. In Proc. of Biometrics Symposium, 2008.

[143] C. Li, Q. Liu, J. Liu, and H. Lu. Learning ordinal discriminative features for age estimation. In Proc. of IEEE Conference on Computer Vision and Pattern Recognition, pages 2570-2577, 2012.

[144] X. Li, S. J. Maybank, S. Yan, D. Tao, and D. Xu. Gait components and their application to gender recognition. IEEE Trans. on System, Man, and Cybernetics Part C, 38(2):145-155, 2008.

[145] D. Lin and X. Tang. From macrocosm to microcosm. In Proc. of IEEE Conference on Computer Vision and Pattern Recognition, pages 1355-1362, 2006.

[146] D.-T. Lin and M.-J. Liu. Face occlusion detection for automated teller machine surveillane. Lecture notes in Computer Science, 4319:641651, 2006.

[147] H. Lin, H. Lu, and L. Zhang. A new automatic recognition system of gender, age and ethnicity. In Proc. of World Congress on Intelligent Control and Automation, volume 2, pages 9988 -9991, 0-0 2006.

[148] S. R. Loth and M.Y. Iscan. Sex determination, Encyclopedia of forensic Sciences, volume 1. Academic Press, San Diego, 2000.

[149] J. Lu, E. Kazmierczak, J. H. Manton, and R. Sinclair. Automatic segmentation of scaling in 2-d psoriasis skin images. IEEE Trans. on Medical Imaging, 32(4):719-730, 2013.

[150] J. Lu and Y.-P. Tan. Gait-based human age estimation. IEEE Trans. on Information Forensics and Security, 2010
[151] X. Lu, H. Chen, and A. K. Jain. Multimodal facial gender and ethnicity identification. In Proc. of International Conference on Biometrics, pages 554-561, 2006.

[152] X. Lu and A. K. Jain. Ethnicity identification from face images. In Proc. of SPIE International Symposium on Defense and Security: Biometric Technology for Human Identification, volume 5404, 2004.

[153] P. Lucey, J.F. Cohn, T. Kanade, J. Saragih, Z. Ambadar, and I. Matthews. The extended cohn-kanade dataset $(\mathrm{ck}+)$ : A complete dataset for action unit and emotion-specified expression. In Proc. of Conference on Computer Vision and Pattern Recognition Workshops, pages 94-101, June 2010.

[154] C. Madden and M. Piccardi. Height measurement as a session-based biometrics for people matching across disjoint camera views. In In Image and Vision Computing New Zealand, page 29, 2005.

[155] M. Madry-Pronobis. Automatic gender recognition based on audiovisual cues. Master Thesis, 2009.

[156] D. Mahajan, S. Sellamanickam, and V. Nair. A joint learning framework for attribute models and object descriptions. In Proc. of International Conference on Computer Vision, 2011.

[157] E. Mäkinen and R. Raisamo. Evaluation of gender classification methods with automatically detected and aligned faces. IEEE Trans. on Pattern Analysis and Machine Intelligence, 2008.

[158] E. Mäkinen and R. Raisamo. An experimental comparison of gender classification methods. Pattern Recognition Letters, 29:1544-1556, 2008.

[159] F. S. Manesh, M. Ghahramani, and Y. P. Tan. Facial part displacement effect on template-based gender and ethnicity classification. In Proc. of International Conference on Control, Automation, Robotics and Vision, pages $1644-1649,2010$

[160] E. Marasco, L. Lugini, and B. Cukic. Exploiting quality and texture features to estimate age and gender from fingerprints. In SPIE Defense+ Security, pages 90750F-90750F, 2014.

[161] E. Marshall. DNA studies challenge the meaning of race. Science, 282:654-655, 1998

[162] A. Martinez and S. Du. A model of the perception of facial expressions of emotion by humans: Research overview and perspectives. The Journal of Machine Learning Research, 13(1):1589-1608, 2012.

[163] A. M. Martinez and R. Benavente. The AR face database. CVC Technical report, (24), 1998.

[164] G. Mather and L. Murdoch. Gender discrimination in biological motion displays based on dynamic cues. In Biological Sciences B, pages 273 279, 1994

[165] J. C. McLachlan and H. Storey. Hot male: can sex in humans be modified by temperature? Journal of theoretical biology, 222:71-72, 2003.

[166] R. Min, A. D'Angelo, and J.-L. Dugelay. Efficient scarf detection prior to face recognition. In Proc. of European Signal Processing Conference, 2010.

[167] R. Min and J. L. Dugelay. Cap detection for moving people in entrance surveillance. In Proc. of ACM Multimedia, 2011.

[168] R. Min, A. Hadid, and J. L. Dugelay. Improving the recognition of faces occluded by facial accessories. In Proc. of IEEE International Conference on Automatic Face and Gesture Recognition, 2011.

[169] B. Moghaddam and M. Yang. Learning gender with support faces. IEEE Trans. on Pattern Analysis and Machine Intelligence, 24:707711, 2002.

[170] Emilio Mordini and Dimitrios Tzovaras. Second generation biometrics: The ethical, legal and social context. Springer, 2012.

[171] M. Moreno-Moreno, J. Fierrez, R. Vera-Rodriguez, and J. Parron. Distance-based feature extraction for biometric recognition of Millimeter Wave body images. In Proc. of IEEE International Carnahan Conference on Security Technology, pages 1-6, Oct 2011.

[172] C. S. Morrison, B. Z. Phillips, J. T. Chang, S. R. Sullivan, and H. O. B Taylor. The relationship between age and facial asymmetry, 2011

[173] K. Moustakas, D. Tzovaras, and G. Stavropoulos. Gait recognition using geometric features and soft biometrics. IEEE Signal processing letters, pages 367-370, 2010.

[174] A. M. Mughal, N. Hassan, and A. Ahmed. Bone age assessment methods: A critical review. Pakistan journal of medical sciences, 30(1):211, 2014

[175] V. Natu, D. Raboy, and A. J. O'Toole. Neural correlates of own-and other-race face perception: Spatial and temporal response differences. NeuroImage, 54(3):2547-2555, 2011.

[176] M. Nazhir, M. Ishaiq, A. Batool, A. Jaffar, and A. M. Mirza. Feature selection for efficient gender classification. In Proc. of International Conference on Neural Networks, Evolutionary Computing and Fuzzy Systems, 2010. 
[177] C. B. Ng, Y. H. Tay, and B.-M. Goi. Vision-based human gender recognition: A survey. Pacific Rim International Conferences on Artificial Intelligence, 7458:335-346, 2012.

[178] B. Ni, Z. Song, and S. Yan. Web image mining towards universal age estimator. In Proc. of ACM Multimedia, 2009.

[179] M. Nixon. Eye spacing measurement for facial recognition. In Proc. of SPIE Applications of Digital Image Processing, volume 575, pages 279-285, 1985.

[180] Mark S. Nixon, Paulo L. Correia, Kamal Nasrollahi, Thomas B. Moeslund, Abdenour Hadid, and Massimo Tistarelli. On soft biometrics. Pattern Recognition Letters, 2015.

[181] A. Nurhudatiana., A. Kong, K. Matinpour, D. Chon, L. Altieri, S. Cho, and N. Craft. The individuality of relatively permanent pigmented or vascular skin marks (RPPVSM) in independently and uniformly distributed patterns. IEEE Trans. on Information Forensics and Security, 2013

[182] E. O. Omidiora, O. Ojo, N. A. Yekini, and T. O. Tubi. Analysis, design and implementation of human fingerprint patterns system. Towards age $\&$ gender determination, ridge thickness to valley thickness ratio (rtvtr) $\&$ ridge count on gender detection. International Journal of Advanced Research in Artificial Intelligence, 1(2):57-63, 2012.

[183] A. Othman and A. Ross. Privacy of facial soft biometrics: Suppressing gender but retaining identity. In Proc. of European Conference on Computer Vision Workshops, 2014.

[184] A. O'Toole, A. Peterson, and K. Deffenbacher. Structural aspects of face recognition and the other race effect. Memory and Cognition, 22:208-224, 1994.

[185] A. O'Toole, A. Peterson, and K. Deffenbacher. An other-race effect for classifying faces by sex. Perception, 25:669-676, 1996.

[186] K. Ozcan, A. K. Mahabalagiri, M. Casares, and S. Velipasalar. Automatic fall detection and activity classification by a wearable embedded smart camera. Emerging and Selected Topics in Circuits and Systems, IEEE Journal on, 3(2):125-136, June 2013.

[187] D. Parikh and K. Grauman. Relative attributes. In Proc. of International Conference on Computer Vision, 2011.

[188] U. Park. Face recognition: face in video, age invariance, and facial marks. Dissertation, 2009

[189] U. Park and A. K. Jain. Face Matching and Retrieval Using Soft Biometrics. IEEE Trans. on Information Forensics and Security, 5(3):406-415, 2010.

[190] U. Park, A. K. Jain, I. Kitahara, K. Kogure, and N. Hagita. Vise: Visual search engine using multiple networked cameras. In Proc. of International Conference on Pattern Recognition, volume 3, pages 1204-1207. IEEE, 2006.

[191] C. Pelin, I. Duyar, E. M. Kayahan, R. Zagyapan, A. M. Agildere, and A. Erar. Body height estimation based on dimensions of sacral and coccygeal vertebrae. Journal of Forensic Sciences, 50(2):294-297, 2005.

[192] P. J. Phillips, F Jiang, A. Narvekar, J. H. Ayyad, and A. J. O'Toole. An other-race effect for face recognition algorithms. ACM Trans. on Applied Perception, 8(2):1-11, 2011.

[193] J. S. Pierrard and T. Vetter. Skin detail analysis for face recognition. In Proc. of IEEE Conference on Computer Vision and Pattern Recognition, pages $1-8,2007$.

[194] S. Prigent, X. Descombes, D. Zugaj, L. Petit, and J. Zerubia. Multiscale analysis of skin hyper-pigmentation evolution. In Proc. of IEEE International Conference on Image Processing, 2013.

[195] H. Proenca, S. Filipe, R. Santos, J. Oliveira, and L. A. Alexandre. The UBIRIS.v2: A Database of Visible Wavelength Iris Images Captured On-The-Move and At-A-Distance. IEEE Trans. on Pattern Analysis and Machine Intelligence, 2009.

[196] M. Pronobis and M. Magimai-Doss. Integrating audio and vision for robust automatic gender recognition. IDIAP Technical Report, IdiapRR-73-2008, 2008.

[197] M. Pronobis and M. Magimai.-Doss. Analysis of F0 and cepstral features for robust automatic gender recognition. IDIAP Technical Report, 2009.

[198] X. Qiu, Z. Sun, and T. Tan. Global texture analysis of iris images for ethnic classification. In Proc. of IAPR International Conference on Biometrics, 2006.

[199] X. Qiu, Z. Sun, and T. Tan. Learning appearance primitives of iris images for ethnic classification. In Proc. of IEEE International Conference on Image Processing, 2007.

[200] N. Ramanathan and R. Chellappa. Modeling age progression in young faces. In Proc. of IEEE Conference on Computer Vision and Pattern Recognition, pages 387-394, 2006.
[201] N. Ramanathan, R. Chellappa, and S. Biswas. Age progression in human faces: A survey. Visual languages and computing, 2009.

[202] V. Ramanathan and H. Wechsler. Robust human authentication using appearance and holistic anthropometric features. Pattern Recognition Letters, 31(15):2425-2435, 2010.

[203] E. Ramón-Balmaseda, J. Lorenzo-Navarro, and M. Castrillón-Santana. Gender classification in large databases. In Progress in Pattern Recognition, Image Analysis, Computer Vision, and Applications, pages 74-81. Springer, 2012.

[204] Y. Ran, Q. Zheng, R. Chellappa, and T. M. Strat. Applications of a simple characterization of human gait in surveillance. IEEE Trans. on System, Man, and Cybernetics Part C, 40(4):1009-1020, 2010.

[205] A. Rattani, C. Chen, and A. Ross. Evaluation of texture descriptors for automated gender estimation from fingerprints. In Proc. of European Conference on Computer Vision Workshops, 2014.

[206] M. H. Raxter, B. M. Auerbach, and C. B. Ruff. Revision of the fully technique for estimating statures. American Journal of Physical Anthropology, 130(3):374-384, 2006.

[207] D. Reid and M. Nixon. Imputing human descriptions in semantic biometrics. In Proc. of ACM Workshop on Multimedia in Forensics, Security and Intelligence, 2010.

[208] D. Reid and M. Nixon. Using comparative human descriptions for soft biometrics. In Proc. of International Joint Conference on Biometrics, 2011.

[209] D. Reid, M. S. Nixon, and S. V. Stevenage. Soft biometrics; human identification using comparative descriptions. IEEE Trans. on Pattern Analysis and Machine Intelligence, PP(99):1-14, 2013.

[210] D. Reid, S. Samangooei, C. Chen, M. Nixon, and A. Ross. Soft biometrics for surveillance: An overview. In C. R. Rao and V. Govindaraju, editors, Handbook of Statistics, volume 31. Elsevier, 2013.

[211] H. T. F. Rhodes. Alphonse Bertillon: Father of scientific detection. Abelard-Schuman, New York, 1956.

[212] M. G. Rhodes. Age estimation of faces: a review. Applications of Cognitive Psychology, 23:112, 2009.

[213] K. Ricanek and B. Barbour. What are soft biometrics and how can they be used? Computer, 44/9:106-108, 2011.

[214] K. Ricanek and T. Tesafaye. Morph: A longitudinal image database of normal adult age-progression. In Proc. of IEEE International Conference on Automatic Face and Gesture Recognition, pages 341345, 2006.

[215] D. Riccio, G. Tortora, M. Marsico, and H. Wechsler. EGA, ethnicity, gender and age, a pre-annotated face database. In Proc. of IEEE Workshop on Biometric Measurements and Systems for Security and Medical Applications, 2012.

[216] A. Rodrigues, J. Sa Silva, and F. Boavida. isenior; A support system for elderly citizens. IEEE Trans. on Emerging Topics in Computing, 1(2):207-217, Dec 2013.

[217] S. M. Mansoor Roomi, S. L. Virasundarii, S. Selvamegala, S. Jeevanandham, and D. Hariharasudhan. Race classification based on facial features. In Proc. of IEEE National Conference on Computer Vision, Pattern Recognition, Image Processing and Graphics, pages 54-57, 2011.

[218] N. A. Rosenberg, J. K. Pritchard, J. L. Weber, H. M. Cann, K. K. Kidd, L. A. Zhivotovsky, and M. W. Feldman. Genetic structure of human populations. Science, 298:2381-2385, 2002.

[219] A. Ross and C. Chen. Can gender be predicted from near-infrared face images? In Proc. of International Conference on Image Analysis and Recognition, 2011.

[220] O. Russakovsky and L. Fei-Fei. Attribute learning in large-scale datasets. In Trends and Topics in Computer Vision, pages 1-14. Springer, 2012

[221] Y. Saatci and C. Town. Cascaded classification of gender and facial expression using active appearance models. In Proc. of IEEE International Conference on Automatic Face and Gesture Recognition, pages 393-400, 2006.

[222] M. Sadeghi, T. K. Lee, D. I. McLean, H. Lui, and M. S. Atkins. Detection and analysis of irregular streaks in dermoscopic images of skin lesions. IEEE Trans. on Medical Imaging, 32(5):849-861, 2013.

[223] S. Samangooei, B. Guo, and Mark S. Nixon. The use of semantic human description as a soft biometric. In Proc. of IEEE International Conference on Biometrics: Theory, Applications and Systems, 2008.

[224] G. Sandbach, S. Zafeiriou, M. Pantic, and L. Yin. Static and dynamic 3D facial expression recognition: A comprehensive survey. Image and Vision Computing, 30(10):683-697, 2012.

[225] S. Sarkar, P. J. Phillips, Z. Liu, I. R. Vega, P. Grother, and K. W Bowyer. The humanid gait challenge problem: Data sets, performance, 
and analysis. IEEE Trans. on Pattern Analysis and Machine Intelligence, 27(2):162-177, 2005.

[226] R. Satta, J. Galbally, and L. Beslay. Children gender recognition under unconstrained conditions based on contextual information. In Proc. of International Conference on Pattern Recognition, 2014.

[227] W. J. Scheirer, N. Kumar, P. N. Belhumeur, and T. E. Boult. Multiattribute spaces: Calibration for attribute fusion and similarity search. In Proc. of IEEE Conference on Computer Vision and Pattern Recognition, pages 2933-2940, 2012.

[228] W. J. Scheirer, N. Kumar, V. N. Iyer, P. N. Belhumeur, and T. E. Boult. How reliable are your visual attributes? In SPIE Defense, Security, and Sensing, pages 87120Q-87120Q, 2013

[229] W. J. Scheirer, N. Kumar, K. Ricanek, P. N. Belhumeur, and T. E. Boult. Fusing with context: a Bayesian approach to combining descriptive attributes. In Proc. of International Joint Conference on Biometrics, 2011.

[230] K. Scherbaum, M. Sunkel, H.-P. Seidel, and V. Blanz. Prediction of individual non-linear aging trajectories of faces. Computer Graphics Forum, 26(3):285-294, 2007.

[231] J. L Scheuer and N. M. Elkington. Sex determination from metacarpals and the first proximal phalanx. Journal of Forensic Sciences, 38:769778, 1993.

[232] H. S. Seung and D. D. Lee. The manifold ways of perception. Science, 290(5500):2268-2269, 2000

[233] L. Shamir. Automatic age estimation by hand photos. Computer Science Letters, 3(1), 2011.

[234] C. Shan. Gender classification on real-life faces. Advanced Concepts for Intelligent Vision Systems, pages 323-331, 2010.

[235] C. Shan. Learning local binary patterns for gender classification on real-world face images. Pattern Recognition Letters, 33(4):431-437, 2012.

[236] C. Shan, S. Gong, and P. W. McOwan. Fusing gait and face cues for human gender recognition. Neurocomputing, 71(10-12):1931-1938, 2008

[237] C. Shan, S. Gong, and P.W. McOwan. Learning gender from human gaits and faces. In Proc. of IEEE International Conference on Advanced Video and Signal based Surveillance, pages 505-510, 2007.

[238] Z. Song, M. Wang, X. Hua, and S. Yan. Predicting occupation via human clothing and contexts. In Proc. of International Conference on Computer Vision, 2011.

[239] V. N. Sorokin and I. S. Makarov. Gender recognition from vocal source. Acoustical Physics, 54(4):571-578, 2008.

[240] N. A. Spaun. Forensic biometrics from images and video at the federal bureau of investigation. In Proc. of IEEE International Conference on Biometrics: Theory, Applications and Systems, 2007.

[241] N. Srinivas, G. Aggarwal, P. J. Flynn, and R. W. Vorder Bruegge. Analysis of facial marks to distinguish between identical twins. IEEE Trans. on Information Forensics and Security, 7(5):1536-1550, Oct 2012

[242] M. Steyn, P. J. Becker, E. N. LAbbé, Y. Scholtz, and J. Myburgh. An assessment of the repeatability of pubic and ischial measurements. Forensic science international, 214(1):210-e1, 2012

[243] K. Sudo, J. Yamato, and A. Tomono. Determining gender of walking people using multiple sensors. In Proc. of IEEE/SICE/RSJ International Conference on Multisensor Fusion and Integration for Intelligent Systems, pages 641-646, 20111996.

[244] N. Sun, W. Zheng, C. Sun, C. Zou, and L. Zhao. Gender classification based on boosting local binary pattern. In: Advances in Neural Networks, pages 194-201, 2006.

[245] Z. Sun, G. Bebis, X. Yuan, and S.J. Louis. Genetic feature subset selection for gender classification: a comparison study. In Proc. of IEEE Workshop on Applications of Computer Vision, pages 165-170, 2002.

[246] J. Suo, S.-C. Zhu, S. Shan, and X. Chen. A compositional and dynamic model for face aging. IEEE Trans. on Pattern Analysis and Machine Intelligence, 32:385-401, 2010.

[247] H. Takimoto, T. Kuwano, Y. Mitsukura, H. Fukai, and M. Fukumi Appearance-age feature extraction from facial image based on age perception. In Proc. of SICE Annual Conference, pages 2813-2818, 2007.

[248] H. Takimoto, Y. Mitsukura, M. Fukumi, and N. Akamatsu. A design of gender and age estimation system based on facial knowledge. In Proc. of SICE International Joint Conference, pages 3883-3886, 2006.

[249] U. Tariq, Yuxiao Hu, and T.S. Huang. Gender and ethnicity identification from silhouetted face profiles. In Proc. of IEEE International Conference on Image Processing, 2009.
[250] J. B. Tenenbaum, V. de Silva, and J. C. Langford. A global geometric framework for nonlinear dimensionality reduction. Science, 290:23192323, 2000.

[251] V. Thomas, N. V. Chawla, K. W. Bowyer, and P. J. Flynn. Learning to predict gender from iris images. In Proc. of IEEE International Conference on Biometrics: Theory, Applications and Systems, 2007.

[252] J. Thornton, J. Baran-Gale, D. Butler, M. Chan, and H. Zwahlen. Person attribute search for large-area video surveillance. In Proc. of IEEE Symposium on Technologies for Homeland Security, pages 5561, 2011.

[253] Q. Tian and S. Chen. Cumulative attribute relation regularization learning for human age estimation. Neurocomputing, 2015.

[254] M. Toews and T. Arbel. Detection, localization, and sex classification of faces from arbitrary viewpoints and under occlusion. IEEE Trans. on Pattern Analysis and Machine Intelligence, 31(9):1567-1581, 2009.

[255] R. J. Tom, T. Arulkumaran, and M. E. Scholar. Fingerprint based gender classification using $2 \mathrm{~d}$ discrete wavelet transforms and principal component analysis. International Journal of Engineering Trends and Technology, 4(2):199-203, 2013.

[256] K. Ueki, T. Hayashida, and T. Kobayashi. Subspace-based age-group classification using facial images under various lighting conditions. In Proc. of IEEE International Conference on Automatic Face and Gesture Recognition, pages 43-48, 2006.

[257] D. Vaquero, R. Feris, D. Tran, L. Brown, A. Hampapur, and M. Turk. Attribute-based people search in surveillance environments. In Proc. of IEEE Workshop on Applications of Computer Vision, 2009.

[258] D. A. Vaquero, R. S. Feris, D. Tran, L. Brown, A. Hampapur, and M. Turk. Attribute-based people search in surveillance environments. In Proc. of Workshop on Applications of Computer Vision, pages 1-8. IEEE, 2009.

[259] C. Velardo, C. Araimo, and J.-L. Dugelay. Synthetic and privacypreserving visualization of video sensor network outputs. In Proc. of ACM / IEEE International Conference on Distributed Smart Cameras 2011.

[260] C. Velardo and J.-L. Dugelay. Weight estimation from visual body appearance. In Proc. of IEEE International Conference on Biometrics: Theory, Applications and Systems, 2010.

[261] C. Velardo, J. L. Dugelay, M. Paleari, and P. Ariano. Building the space scale or how to weigh a person with no gravity. In Proc. of IEEE International Conference on Emerging Signal Processing Applications, 2012.

[262] P. Viola, G. Shakhnarovich, G. Shakhnarovich, P. A. Viola, and B. Moghaddam. A unified learning framework for real time face detection and classification. In Proc. of IEEE International Conference on Automatic Face and Gesture Recognition, 2002.

[263] T. Wadhawan, N. Situ, H. Rui, K. Lancaster, X. Yuan, and G. Zouridakis. Implementation of the 7-point checklist for melanoma detection on smart handheld devices. In Proc. of International Conference of the IEEE in Medicine and Biology Society, pages 3180-3183, 2011.

[264] L. Walawalkar, M. Yeasin, A. M. Narasimhamurthy, and R. Sharma. Support vector learning for gender classification using audio and visual cues: A comparison. In Proc. of SVM, pages 144-159, 2002.

[265] J. Wang, J. Li, W. Yau, and E. Sung. Boosting dense SIFT descriptors and shape contexts of face images for gender recognition. In Proc. of Conference on Computer Vision and Pattern Recognition Workshops, pages 96-102, 2010.

[266] S. Wang, M. Skubic, and Y. Zhu. Activity density map visualization and dissimilarity comparison for eldercare monitoring. Information Technology in Biomedicine, IEEE Trans. on, 16(4):607-614, July 2012

[267] X. Wang, R. Guo, and C. Kambhamettu. Deeply-learned feature for age estimation. In Proc. of Winter Conference on Applications of Computer Vision, pages 534-541. IEEE, 2015.

[268] C. I. Watson. Mugshot identification data - fronts and profiles. In Reference Data of NIST Special Database, 1994.

[269] C.-Y. Wen, S.-H. Chiu, Y.-R. Tseng, and C.-P. Lu. The mask detection technology for occluded face analysis in the surveillance system. Journal of Forensic Sciences, 50(3):593-601, 2005.

[270] L. Wen and G-D. Guo. A computational approach to body mass index prediction from face images. Image and Vision Computing, 31(5):392400, 2013.

[271] D. A. Wilbraham, J. C. Christensen, A. M. Martinez, and J. T. Todd. Can low level image differences account for the ability of human observers to discriminate facial identity? Journal of Vision, 8(15):5, 2008.

[272] H. Wu, G. Yoshikawa, T. Shioyama, S. Lao, and M. Kawade. Glasses frame detection with 3D Hough transform. In Proc. of International Conference on Pattern Recognition, volume 16, pages 346-349, 2002. 
[273] K. Wu and D. G. Childers. Gender recognition from speech. part I: Coarse analysis. Journal of the Acoustical Society of America, 90:1828-1840, 1991

[274] B. Xia, B. Ben Amor, M. Daoudi, and H. Drira. Can 3D shape of the face reveal your age? In Proc. of International Conference on Computer Vision Theory and Applications, 2014.

[275] B. Xia, H. Sun, and B.-L. Lu. Multi-view gender classification based on local gabor binary mapping pattern and support vector machines. In Proc. of International Joint Conference on Neural Networks , pages 3388-3395, 2008.

[276] B. Xiao, X. Yang, and Y. Xu. Learning distance metric for regression by semidefinite programming with application to human age estimation. In Proc. of ACM Multimedia, 2009.

[277] Y. Xiao and H. Yan. Extraction of glasses in human face images. In Proc. of International Conference on Biometric Authentication, 2004.

[278] Y. Xie, K. Luu, and M. Savvides. A robust approach to facial ethnicity classification on large scale face databases. In Proc. of BTAS, pages 143-149, Sept 2012.

[279] S. Yan, H. Wang, Y. Fu, J. Yan, X. Tang, and T. S. Huang. Synchronized submanifold embedding for person independent pose estimation and beyond. IEEE Trans. on Image Processing, 18(1):202-210, 2009.

[280] S. Yan, D. Xu, B. Zhang, H. Zhang, Q. Yang, and S. Lin. Graph embedding and extension: a general framework for dimensionality reduction. IEEE Trans. on Pattern Analysis and Machine Intelligence, 29(1):40-51, 2007.

[281] Z. Yang and H. Ai. Demographic classification with local binary pattern. In Proc. of IAPR International Conference on Biometrics, 2007.

[282] E. A. Yates, A. K. Macpherson, and J. L. Kuk. Secular trends in the diagnosis and treatment of obesity among us adults in the primary care setting. Obesity, 20(9):1909-1914, 2012.

[283] J. Yoo, D. Hwang, and M. S. Nixon. Gender classification in human gait with svm. Advanced concepts for intelligent vision systems, 3708:138145,2005

[284] S. M. Yoon and S. C. Kee. Detection of partially occluded face using support vector machines. In Proc. of IAPR MVA, 2002.

[285] S. Yu, D. Tan, and T. Tan. A framework for evaluating the effect of view angle, clothing and carrying condition on gait recognition. In Proc. of International Conference on Pattern Recognition, 2006.

[286] S. Yu, T. Tan, K. Huang, K. Jia, and X. Wu. A study on gait-based gender classification. IEEE Trans. on Image Processing, 18(8):19051910, 2009.

[287] J. C. Yuille and J. L. Cutshall. A case study of eyewitness memory of a crime. Journal of applied psychology, 71(2):291-301, 1986.

[288] Z. Zeng, M. Pantic, G. I. Roisman, and T. S. Huang. A survey of affect recognition methods: Audio, visual, and spontaneous expressions. IEEE Trans. on Pattern Analysis and Machine Intelligence, 31(1):3958, 2009.

[289] R. Zewail, A. Elsafi, M. Saeb, and N. Hamdy. Soft and hard biometrics fusion for improved identity verification. In Proc. of IEEE International Midwest Symposium on Circuits and Systems, volume 1, pages I - 2258, 2004.

[290] D. Zhang, Y. Wang, and Z. Zhang. Ethnicity classification based on a hierarchical fusion. In W.-S. Zheng, Z. Sun, Y. Wang, X. Chen, P. C. Yuen, and J. Lai, editors, Biometric Recognition, volume 7701 of Lecture Notes in Computer Science, pages 300-307. Springer Berlin Heidelberg, 2012.

[291] D. Zhang, Y. Wang, Z. Zhang, and M. Hu. Ethnicity classification based on fusion of face and gait. In Proc. of International Conference on Biometrics, pages 384-389, 2012.

[292] D. Zhang, Q. Zhao, and F. Chen. Quantitative analysis of human facial beauty using geometric features. Pattern Recognition, 44(4):940 - 950, 2011.

[293] G. Zhang and Y. Wang. Multimodal 2D and 3D facial ethnicity classification. In Proc. of International Conference on Image and Graphics, 2009.

[294] H. Zhang, Z. Sun, T. Tan, and J. Wang. Ethnic classification based on iris images. In Z. Sun, J. Lai, X. Chen, and T. Tan, editors, Biometric Recognition, volume 7098 of Lecture Notes in Computer Science, pages 82-90. 2011.

[295] Z. Zhang. A flexible new technique for camera calibration. IEEE Trans. on Pattern Analysis and Machine Intelligence, 22(11):13301334, 2000.

[296] S. K. Zhou, B. Georgescu, X. S. Zhou, and D. Comaniciu. Image based regression using boosting method. In Proc. of International Conference on Computer Vision, pages 541-548, 2005.
[297] Z. Zhou, Y. Wang, and E. K. Teoh. People re-identification based on bags of semantic features. In Proc. of Asian Conference on Computer Vision Workshops, pages 574-586. Springer, 2014.

[298] X. Zhuang, X. Zhou, M. Hasegawa-Johnson, and T. S. Huang. Face age estimation using patch-based hidden markov model supervectors. In Proc. of International Conference on Pattern Recognition, 2008.

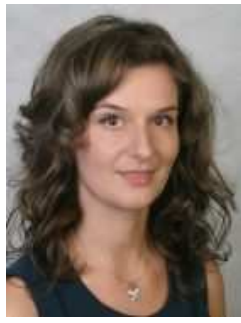

Antitza Dantcheva is a Marie Curie Post-Doctora fellow at the STARS team, INRIA, France. Previously, she was a Post-Doctoral fellow at the Michigan State University and the West Virginia University, USA. She received her PhD in Signal and Image Processing in 2011 from Eurecom / Telecom ParisTech in France. She was the recipient of the Best Presentation Award in ICME 2011, the Best Poster Award in ICB 2013 as well as the Tabula Rasa Spoofing Award in ICB 2013. Her research interests are in soft biometrics for security and commercial applications, where she has worked on retrieval of soft biometrics from images, as well as their corresponding analysis.

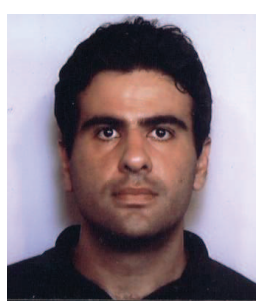

Petros Elia is an Assistant Professor with the Department of Mobile Communications at EURECOM in Sophia Antipolis, France. He received the B.Sc. degree from the Illinois Institute of Technology, and the M.Sc. and Ph.D. degrees in electrical engineering from the University of Southern California (USC), Los Angeles, in 2001 and 2006 respectively. Since 2008 he has been at EURECOM.

His latest research deals with the role of feedback and complexity in multiuser communications, MIMO, cooperative and multiple access protocols and transceivers, complexity of communication, as well as with isolation and connectivity in dense networks, queueing theory and cross-layer design, coding theory, information theoretic limits in cooperative communications, and surveillance networks. He is a Fulbright scholar, the co-recipient of the SPAWC-2011 best student paper award on the topic of reduced complexity bidirectional communication with limited feedback, and of the NEWCOM++ distinguished achievement award 2008-2011 for a sequence of publications on the topic of reduced complexity multimode communications in the presence of little or no feedback.

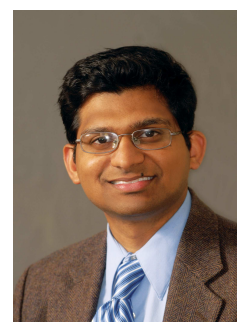

Arun Ross is an Associate Professor in the Department of Computer Science and Engineering at Michigan State University. He received the B.E. (Hons.) degree in Computer Science from the Birla Institute of Technology and Science, Pilani, India, in 1996, and the M.S. and Ph.D. degrees in Computer Science and Engineering from Michigan State University, East Lansing, in 1999 and 2003, respectively. Between 1996 and 1997, he was with the Design and Development Group of Tata Elxsi (India) Ltd., Bangalore, India. He also spent three summers (2000 - 2002) with the Imaging and Visualization Group of Siemens Corporate Research, Inc., Princeton, NJ, working on fingerprint recognition algorithms. His research interests include pattern recognition, classifier fusion, computer vision, and biometrics. He is the coauthor of the books "Introduction to Biometrics: A Textbook" and "Handbook of Multibiometrics", and the coeditor of "Handbook of Biometrics". Arun is a recipient of NSF's CAREER Award and was designated a Kavli Frontier Fellow by the National Academy of Sciences in 2006. He was an Associate Editor of the IEEE Transactions on Image Processing and the IEEE Transactions on Information Forensics and Security. 